\title{
THE BAKER-CAMPBELL-HAUSDORFF FORMULA AND THE ZASSENHAUS FORMULA IN SYNTHETIC DIFFERENTIAL GEOMETRY
}

\author{
HIROKAZU NISHIMURA
}

\begin{abstract}
After the torch of Anders Kock [6], we will establish the Baker-CampbellHausdorff formula as well as the Zassenhaus formula in the theory of Lie groups.
\end{abstract}

\section{INTRODUCTION}

The Baker-Campbell-Hausdorff formula (the BCH formula for short) was first discovered by Campbell ([2] and [3]) on the closing days of the 19th century so as to construct a Lie group directly from a given Lie algebra (i.e., Lie's third fundamental theorem !). However, his investigation failed in convergence problems, let alone dealing only with matrix Lie algebras. The $\mathrm{BCH}$ formula was finally established by Baker [1] and Hausdorff [5] independently within a somewhat more abstract framework of formal power series on the dawning days of the 20th century, getting rid of convergence problems completely while losing touch with the theory of Lie groups. The $\mathrm{BCH}$ formula resurrected its touch with the theory of Lie groups thanks to Magnus [9] in the middle of the 20th century.

The BCH formula claims, roughly speaking, that the multiplication in a Lie group is already encoded in its Lie algebra. More precisely, the multiplication in a Lie group is expressible in terms of Lie brackets in its Lie algebra, which readily gives rise to Lie's second fundamental theorem in the theory of finite-dimensional Lie groups, though the modern treatment of the theory of finite-dimensional Lie groups is liable to base Lie's second fundamental theorem somewhat opaquely upon the Frobenius theorem.

The so-called Taylor formula was introduced by the English mathematician called Brook Taylor in the early 18th century, though its pedigree can be traced back even to Zeno in ancient Greece. Kock [6] has shown that the nature of the Taylor formula in differential calculus is more combinatorial or algebraic than analytical, dodging convergence problems completely, as far as we are admitted to speak on the infinitesimal level, where nilpotent infinitesimals are available in plenty. The principal objective of this paper is to do the same with the $\mathrm{BCH}$ formula and its inverse companion called the Zassenhaus formula in the theory of Lie groups, though we must confront the noncommutative world in sharp contrast

$M S C$ (2010): primary 51K10, 17-08, 17B01, 22E60.

Keywords: synthetic differential geometry, the Baker-Campbell-Hausdorff formula, the Zassenhaus formula, logarithmic derivative. 
to the Taylor formula living a commutative life. We have found out that the Zassenhaus formula is much easier to deal with than the $\mathrm{BCH}$ formula itself, albeit, historically speaking, the former having been found out by Zassenhaus [18] within an abstract framework of formal power series more than three decades later than the latter and its continuous counterpart having been established by Fer [4] four years later than [9]. Strangely enough, our BCH formula diverges from the usual one in the 4-th order. The $\mathrm{BCH}$ formula will be dealt with in $\S 7$ and $\S 8$ by two different methods, while we will be concerned with the Zassenhaus formula in $\S 6$. We approach the $\mathrm{BCH}$ formula in anticipation of its validity in $\S 7$ by using only the left logarithmic derivative of the exponential mapping, while we will do so from scratch in $\S 8$ by using both the left and right logarithmic derivatives of the exponential mapping. As expected, the latter proofs are longer than the former ones.

We will work within the framework of synthetic differential geometry as in [8]. We assume the reader to be familiar with Chapters 1-3 of [8]. Now we fix our terminology and notation. Given a microlinear space $M$, we denote $M^{D}$ by $\mathbf{T} M$, while we denote the tangent space of $M$ at $x \in M$ by $\mathbf{T}_{x} M=\{\gamma \in \mathbf{T} M \mid \gamma(0)=x\}$. Given a mapping $f: M \rightarrow N$ of microlinear spaces, its differential is denoted by $\mathbf{d} f$, which is a mapping from $\mathbf{T} M$ to $\mathbf{T} N$, assigning $f \circ \gamma \in \mathbf{T} N$ to each $\gamma \in \mathbf{T} M$. We denote the identity mapping of $M$ by $\operatorname{id}_{M}$. The unit element of a group $G$ is usually denoted by $e$. In the proof of a theorem or the like, we insert some comment surrounded with parentheses )(.

\section{The Lie Algebra of A Lie group}

Definition 2.1. A Lie group is a group which is microlinear as a space.

Notation 2.2. Given a Lie group $G$, its tangent space $\mathbf{T}_{e} G$ at $e$ is usually denoted by its corresponding German letter $\mathfrak{g}$.

From now on, $G$ will always be assumed to be a Lie group with $\mathfrak{g}=\mathbf{T}_{e} G$.

Proposition 2.3. Given $X \in \mathfrak{g}$ and $\left(d_{1}, d_{2}\right) \in D(2)$, we have

$$
X_{d_{1}+d_{2}}=X_{d_{1}} \cdot X_{d_{2}} \text {. }
$$

Proof. By the same argument as in Proposition 3, §3.2 of [8].

\section{Corollary 2.4.}

$$
X_{-d}=\left(X_{d}\right)^{-1}
$$

Proof. Evidently

$$
(d,-d) \in D(2)
$$

is obtained, so that we get

$$
e=X_{d+(-d)}=X_{d} \cdot X_{-d}=X_{-d} \cdot X_{d}
$$

by the above proposition.

Proposition 2.5. Given $X, Y \in \mathfrak{g}$ and $d \in D$, we have

$$
(X+Y)_{d}=X_{d} \cdot Y_{d}=Y_{d} \cdot X_{d}
$$


Proof. By the same argument as in Proposition 6, §3.2 of [8].

Theorem 2.6. Given $X, Y \in \mathfrak{g}$, there exists a unique $Z \in \mathfrak{g}$ with

$$
X_{d_{1}} \cdot Y_{d_{2}} \cdot X_{-d_{1}} \cdot Y_{-d_{2}}=Z_{d_{1} d_{2}}
$$

for any $d_{1}, d_{2} \in D$.

Proof. By the same argument as in pp. 71-72 of [8].

Definition 2.7. We denote $Z$ in the above theorem by $[X, Y]$, so that we have a function

$$
[\cdot, \cdot]: \mathfrak{g} \times \mathfrak{g} \rightarrow \mathfrak{g}
$$

called the Lie bracket.

Theorem 2.8. The $\mathbb{R}$-module $\mathfrak{g}$ endowed with the Lie bracket $[\cdot, \cdot]: \mathfrak{g} \times \mathfrak{g} \rightarrow \mathfrak{g}$ is a Lie algebra.

Proof. By the same argument as in Proposition 7 (§3.2) of [8].

Proposition 2.9. Given a homomorphism

$$
\varphi: G \rightarrow H
$$

of Lie groups, the mapping

$$
\varphi^{\prime}: \mathfrak{g} \rightarrow \mathfrak{h}
$$

obtained as the restriction of the differential

$$
\mathbf{d} \varphi: \mathbf{T} G \rightarrow \mathbf{T} H
$$

to $\mathfrak{g}=\mathbf{T}_{e} G$ is a homomorphism of Lie algebras.

Proof. Given $X, Y \in \mathfrak{g}$ and $d_{1}, d_{2} \in D$, we have

$$
\begin{aligned}
& (\mathbf{d} \varphi([X, Y]))_{d_{1} d_{2}} \\
= & \varphi\left([X, Y]_{d_{1} d_{2}}\right) \\
= & \varphi\left(X_{d_{1}} \cdot Y_{d_{2}} \cdot X_{-d_{1}} \cdot Y_{-d_{2}}\right) \\
= & \varphi\left(X_{d_{1}}\right) \cdot \varphi\left(Y_{d_{2}}\right) \cdot \varphi\left(X_{-d_{1}}\right) \cdot \varphi\left(Y_{-d_{2}}\right) \\
= & (\mathbf{d} \varphi(X))_{d_{1}} \cdot(\mathbf{d} \varphi(Y))_{d_{2}} \cdot(\mathbf{d} \varphi(X))_{-d_{1}} \cdot(\mathbf{d} \varphi(Y))_{-d_{2}} \\
= & {[\mathbf{d} \varphi(X), \mathbf{d} \varphi(Y)]_{d_{1} d_{2}} }
\end{aligned}
$$

so that $\varphi^{\prime}$ preserves Lie brackets.

The next simple lemma will be useful in the last section.

Lemma 2.10. Given $X, Y \in \mathfrak{g}$, we have

$$
[X,[Y,[X, Y]]]=[Y,[X,[X, Y]]] .
$$

Proof. This follows easily from the following Jacobi identity:

$$
[X,[Y,[X, Y]]]+[Y,[[X, Y], X]]+[[X, Y],[X, Y]]=0 .
$$


Notation 2.11. Given a Euclidean $\mathbb{R}$-module $V$ which is microlinear as a space, the totality of bijective homomorphisms of $\mathbb{R}$-modules from $V$ onto itself is denoted by $G L(V)$, which is a Lie group with composition of mappings as its group operation (cf. Proposition 5 (§§3.2) of [8]). Its Lie algebra is usually denoted by $\mathfrak{g l}(V)$.

Proposition 2.12. Given a Euclidean $\mathbb{R}$-module $V$ which is microlinear as a space, the Lie algebra $\mathfrak{g l}(V)$ can naturally be identified with the Lie algebra of homomorphisms of $\mathbb{R}$-modules from $V$ into itself with its Lie bracket

$$
[\varphi, \psi]=\varphi \circ \psi-\psi \circ \varphi
$$

for any homomorphisms $\varphi, \psi$ of $\mathbb{R}$-modules from $V$ into itself.

Proof. Given a mapping $X: D \rightarrow G L(V)$ with $X_{0}=\mathrm{id}_{V}$, there exists a unique mapping $\varphi: V \rightarrow V$ such that

$$
X_{d}(u)=u+d \varphi(u)
$$

for any $d \in D$ and any $u \in V$, since the $\mathbb{R}$-module $V$ is Euclidean by assumption. Since $X_{d} \in G L(V)$, we have

$$
\alpha u+d \varphi(\alpha u)=X_{d}(\alpha u)=\alpha X_{d}(u)=\alpha u+\alpha d \varphi(u)
$$

for any $\alpha \in \mathbb{R}$, any $u \in V$ and any $d \in D$, so that we get

$$
\varphi(\alpha u)=\alpha \varphi(u)
$$

for any $\alpha \in \mathbb{R}$ and any $u \in V$, which implies that the mapping $\varphi: V \rightarrow V$ is a homomorphism of $\mathbb{R}$-modules (cf. Proposition 10 ( $\S 1.2)$ in [8]). Conversely, given a homomorphism $\varphi$ of $\mathbb{R}$-modules from $V$ into itself and $d \in D, \operatorname{id}_{V}+d \varphi$ is obviously a homomorphism of $\mathbb{R}$-modules from $V$ into itself, and we have

$$
\left(\mathrm{id}_{V}+d \varphi\right) \circ\left(\mathrm{id}_{V}-d \varphi\right)=\left(\mathrm{id}_{V}-d \varphi\right) \circ\left(\mathrm{id}_{V}+d \varphi\right)=\mathrm{id}_{V}
$$

so that the mapping $\operatorname{id}_{V}+d \varphi$ is bijective. Therefore we are sure that the $\mathbb{R}$-module $\mathfrak{g l}(V)$ is naturally identified with the $\mathbb{R}$-module of homomorphisms of $\mathbb{R}$-modules from $V$ into itself. It remains to be shown that this identification preserves Lie brackets. Let us assume that $X \in \mathfrak{g l}(V)$ corresponds to the homomorphism $\varphi$ of $\mathbb{R}$-modules from $V$ into itself, while $Y \in \mathfrak{g l}(V)$ corresponds to the homomorphism $\psi$ of $\mathbb{R}$-modules from $V$ into itself. Then, given $d_{1}, d_{2} \in D$, we have

$$
\begin{aligned}
& {[X, Y]_{d_{1} d_{2}} } \\
= & X_{d_{1}} \cdot Y_{d_{2}} \cdot X_{-d_{1}} \cdot Y_{-d_{2}} \\
= & \left(\operatorname{id}_{V}+d_{1} \varphi\right) \circ\left(\operatorname{id}_{V}+d_{2} \psi\right) \circ\left(\operatorname{id}_{V}-d_{1} \varphi\right) \circ\left(\operatorname{id}_{V}-d_{2} \psi\right) \\
= & \left\{\operatorname{id}_{V}+d_{1} \varphi+d_{2} \psi+d_{1} d_{2} \varphi \circ \psi\right\} \circ\left\{\operatorname{id}_{V}-d_{1} \varphi-d_{2} \psi+d_{1} d_{2} \varphi \circ \psi\right\} \\
= & \operatorname{id}_{V}-d_{1} \varphi-d_{2} \psi+d_{1} d_{2} \varphi \circ \psi+d_{1} \varphi-d_{1} d_{2} \varphi \circ \psi+d_{2} \psi-d_{1} d_{2} \psi \circ \varphi+d_{1} d_{2} \varphi \circ \psi \\
= & \operatorname{id}_{V}+d_{1} d_{2}(\varphi \circ \psi-\psi \circ \varphi)
\end{aligned}
$$

so that our identification of $\mathfrak{g l}(V)$ with the $\mathbb{R}$-module of homomorphisms of $\mathbb{R}$ modules from $V$ into itself indeed preserves Lie brackets. 


\section{The ADJOInt RePRESEntations}

Notation 3.1. Given $x \in G$, the mapping $y \in G \mapsto x y x^{-1} \in G$ is obviously a homomorphism of groups, naturally giving rise to a mapping $\mathfrak{g} \rightarrow \mathfrak{g}$ as derivative, which we denote by $\operatorname{Ad} x \in G L(\mathfrak{g})$. Thus we have a homomorphism Ad $: G \rightarrow$ $G L(\mathfrak{g})$ of groups, naturally giving rise to a mapping ad $: \mathfrak{g} \rightarrow \mathfrak{g l}(\mathfrak{g})$ as derivative.

Theorem 3.2. Given $X, Y \in \mathfrak{g}$, we have

$$
(\operatorname{ad} X)(Y)=[X, Y] .
$$

Proof. Given $d, d^{\prime} \in D$, we have

$$
\begin{aligned}
& \left(\left(\operatorname{Ad} X_{d}\right)(Y)-Y\right)_{d^{\prime}}=X_{d} \cdot Y_{d^{\prime}} \cdot X_{-d} \cdot Y_{-d^{\prime}} \\
& ) \text { By Proposition } 2.5( \\
= & {[X, Y]_{d d^{\prime}}=(d[X, Y])_{d^{\prime}} }
\end{aligned}
$$

so that we have the desired formula.

\section{The exponential mapping}

Our notions of a one-parameter subgroup, a left-invariant vector field, etc. are standard, and it is easy to see that

Proposition 4.1. Given a mapping $\theta: \mathbb{R} \rightarrow G$, the following conditions are equivalent:

(1) The mapping $\theta: \mathbb{R} \rightarrow G$ is a one-parameter subgroup.

(2) The mapping $\theta: \mathbb{R} \rightarrow G$ is a flow of a left invariant vector field on $G$ with $\theta(0)=e$.

(3) The mapping $\theta: \mathbb{R} \rightarrow G$ is a flow of a right invariant vector field on $G$ with $\theta(0)=e$.

Notation 4.2. Given $X \in \mathfrak{g}$, if there is a one-parameter subgroup $\theta: \mathbb{R} \rightarrow G$ with $\mathbf{d} \theta\left(i_{D}^{\mathbb{R}}\right)=X$, then we write $\exp ^{G} X$ or $\exp X$ for $\theta(1)$.

The following definition is borrowed from 38.4 in [7], which is, in turn, owing to the research [11]-[16] of Omori et al.

Definition 4.3. A Lie group $G$ is called regular provided that, for any mapping $\varsigma: \mathbb{R} \rightarrow \mathfrak{g}$, there exists a mapping $\theta: \mathbb{R} \rightarrow G$ with

$$
\theta(0)=e
$$

and

for any $t \in \mathbb{R}$ and any $d \in D$.

$$
\theta(t+d)=\theta(t) \cdot \varsigma(t)_{d}
$$

From now on, we will assume the Lie group $G$ to be regular, so that $\exp ^{G}: \mathfrak{g} \rightarrow$ $G$ is indeed a total function.

Notation 4.4. Given $\xi \in \mathfrak{g l}(V)$ with $\xi^{n+1}$ vanishing for some natural number $n$, we write

$$
e^{\xi}=\sum_{i=0}^{n} \frac{\xi^{i}}{i !} .
$$


It is easy to see that

Lemma 4.5. Given $\xi \in \mathfrak{g l}(V)$ with $\xi^{n+1}$ vanishing for some natural number $n$, we have

$$
\exp ^{G L(V)} \xi=e^{\xi}
$$

Proposition 4.6. Given a homomorphism $\varphi: G \rightarrow H$ of Lie groups and $X \in \mathfrak{g}, \exp ^{H} \varphi^{\prime}(X)$ is defined, and we have

$$
\exp ^{H} \varphi^{\prime}(X)=\varphi\left(\exp ^{G} X\right) .
$$

Remark 4.7. The Lie group $G$ is assumed to be regular, as we have said before, but the Lie group $H$ is not assumed to be regular, so that $\exp ^{H}$ is not necessarily a total function.

Proof. It suffices to note that, given a one-parameter subgroup $\theta: \mathbb{R} \rightarrow G$ of $G$ with

$$
\mathbf{d} \theta\left(i_{D}^{\mathbb{R}}\right)=X
$$

the mapping $\varphi \circ \theta: \mathbb{R} \rightarrow H$ is a one-parameter subgroup of $H$ with

$$
\mathbf{d}(\varphi \circ \theta)\left(i_{D}^{\mathbb{R}}\right)=\varphi^{\prime}(X) .
$$

Proposition 4.8. Given $X \in \mathfrak{g}$ with $(\operatorname{ad} X)^{n+1}$ vanishing for some natural number n, we have

$$
\operatorname{Ad}(\exp X)=e^{\operatorname{ad} X} \text {. }
$$

Proof. We have

$$
\begin{aligned}
& \operatorname{Ad}\left(\exp ^{G} X\right)=\exp ^{G L(V)}(\operatorname{ad} X) \\
& \text { By Proposition } 4.6( \\
= & e^{\operatorname{ad} X}
\end{aligned}
$$

)By Lemma 4.5(

We conclude this section by the following simple but significant proposition.

Proposition 4.9. We have

$$
\exp t(d X)=X_{t d}
$$

for any $t \in \mathbb{R}$. In particular, we have

$$
\exp d X=X_{d}
$$

by setting $t=1$.

Proof. For any $d^{\prime} \in D$, we have

$$
\begin{aligned}
& (d X)_{t+d^{\prime}}=X_{\left(t+d^{\prime}\right) d}=X_{t d+d^{\prime} d}=X_{t d} \cdot X_{d^{\prime} d} \\
& ) \text { By Proposition } 2.3( \\
= & (d X)_{t} \cdot(d X)_{d^{\prime}}
\end{aligned}
$$

so that we have the desired conclusion. 


\section{LOGARITHMiC DERIVATIVES}

In this section we deal with the left and right derivatives. First we deal with the left derivative.

Definition 5.1. Given a microlinear space $M$ and a function $f: M \rightarrow G$, the function

$$
\delta^{\mathrm{left}} f: \mathbf{T} M \rightarrow \mathfrak{g}
$$

is defined to be such that

$$
(\mathbf{d} f(X))_{d}=f(x) \cdot\left(\delta^{\text {left }} f(X)\right)_{d}
$$

for any $x \in M$, any $X \in \mathbf{T}_{x} M$ and any $d \in D$. It is called the left logarithmic derivative of $f$. The restriction of $\delta^{\text {left }} f$ to $\mathbf{T}_{x} M$ is denoted by $\delta^{\text {left }} f(x)$.

The following is the Leibniz rule for the left logarithmic derivative.

Proposition 5.2. Let $M$ be a microlinear space. Given two functions $f, g$ : $M \rightarrow G$ together with $X \in \mathbf{T} M$, we have

$$
\delta^{\text {left }}(f g)(X)=\delta^{\text {left }} g(X)+\operatorname{Ad}\left(g(x)^{-1}\right)\left(\delta^{\text {left }} f(X)\right)
$$

with $x=X_{0}$.

Proof. For any $d \in D$, we have

$$
\begin{aligned}
& \left(\delta^{\text {left }}(f g)(X)\right)_{d} \\
= & g(x)^{-1} \cdot f(x)^{-1} \cdot f\left(X_{d}\right) \cdot g\left(X_{d}\right) \\
= & g(x)^{-1} \cdot f(x)^{-1} \cdot f\left(X_{d}\right) \cdot g(x) \cdot g(x)^{-1} \cdot g\left(X_{d}\right) \\
= & \left\{\operatorname{Ad}\left(g(x)^{-1}\right)\left(\delta^{\text {left }} f(X)\right)+\delta g(X)\right\}_{d} \\
& \text { )By Proposition } 2.5(
\end{aligned}
$$

so that we get the desired formula.

Theorem 5.3. Given $X \in \mathfrak{g}$ with $(\operatorname{ad} X)^{n+1}$ vanishing for some natural number $n$, we have

$$
\delta^{\text {left }}(\exp )(X)=\sum_{p=0}^{n} \frac{(-1)^{p}}{(p+1) !}(\operatorname{ad} X)^{p} .
$$

Proof. The proof is essentially along the lines of Lemma 4.27 of [10]. We have

$$
\begin{aligned}
& (s+t) \delta^{\text {left }}(\exp )((s+t) X) \\
= & \delta^{\text {left }}(\exp (s+t) \cdot)(X) \\
& ) \text { By the chain rule of differentiation( } \\
= & \delta^{\text {left }}((\exp s \cdot)(\exp t \cdot))(X) \\
= & \delta^{\text {left }}(\exp t \cdot)(X)+\operatorname{Ad}(\exp (-t) X)\left(\delta^{\text {left }}(\exp s \cdot)\right)(X) \\
& ) \text { By Proposition } 5.2( \\
= & t \delta^{\text {left }}(\exp )(t X)+\operatorname{Ad}(\exp (-t) X)\left(s \delta^{\text {left }}(\exp )(s X)\right)
\end{aligned}
$$


so that, by letting

$$
F(s)=s \delta^{\text {left }}(\exp )(s X)
$$

so as to introduce a function $F: \mathbb{R} \rightarrow L(\mathfrak{g}, \mathfrak{g})$, we get

$$
F(s+t)=F(t)+\operatorname{Ad}(\exp (-t) X)(F(s)),
$$

which earns us

$$
F^{\prime}(s)=F^{\prime}(0)-(\operatorname{ad} X)(F(s))
$$

by fixing $s$ and differentiaing with respect to $t$ at $t=0$. Since we have also

$$
F^{\prime}(s)=\delta^{\text {left }}(\exp )(s X)+s \delta^{\text {left }}(\exp )(X)
$$

we get

$$
F^{\prime}(0)=\mathrm{id}_{\mathfrak{g}},
$$

by letting $s=0$, so that the formula (5.1) is transmogrified into the ordinary differential equation

$$
F^{\prime}(s)=\operatorname{id}_{\mathfrak{g}}-(\operatorname{ad} X)(F(s))
$$

on $L(\mathfrak{g}, \mathfrak{g})$. Its unique solution with the initial condition of $F(0)$ 's vanishing is

$$
F(s)=\sum_{p=0}^{n} \frac{(-1)^{p} s^{p+1}}{(p+1) !}(\operatorname{ad} X)^{p},
$$

which results in the desired formula by letting $s=1$.

Proposition 5.4. Given $X, Y \in \mathfrak{g}$ with $[X, Y]$ vanishing, we have

$$
\exp X \cdot \exp Y=\exp X+Y \text {. }
$$

In particular, we have

$$
\exp X \cdot \exp Y=\exp Y \cdot \exp X .
$$

Proof. Letting $H(t)=\exp X . \exp t Y$. exp $-(X+t Y)$ so as to get a function $H: \mathbb{R} \rightarrow G$, we have $H(0)=e$ evidently. By differentiating $H$ logarithmically, we have

$$
\begin{aligned}
& \delta^{\text {left }} H(t) \\
= & \delta^{\text {left }}(\exp )(-(X+t Y))(-Y)+\operatorname{Ad}(\exp X+t Y)\left(\delta^{\text {left }}(\exp )(t Y)(Y)\right) \\
= & -Y+\operatorname{Ad}(\exp X+t Y)(Y)=-Y+e^{\operatorname{ad}(X+t Y)}(Y)=-Y+Y=0
\end{aligned}
$$

so that we have the desired formula.

Proposition 5.5. Given $X, Y \in \mathfrak{g}$ and $d_{1}, d_{2} \in D$, we have

$$
\exp d_{1} X . \exp d_{2} Y=\exp d_{2} Y \cdot \exp d_{1} X . \exp d_{1} d_{2}[X, Y] \text {. }
$$

Proof. We have

$$
\begin{aligned}
& \exp d_{1} X+d_{2} Y=\exp d_{1} X .\left\{\delta^{\text {left }}(\exp )\left(d_{1} X\right)(Y)\right\}_{d_{2}} \\
& \text { logarithmic derivative }( \\
= & \exp d_{1} X \cdot\left\{Y-\frac{1}{2} d_{1}[X, Y]\right\}_{d_{2}}
\end{aligned}
$$

)By Theorem 5.3( 


$$
=\exp d_{1} X . Y_{d_{2}} \cdot\left(-\frac{1}{2} d_{1}[X, Y]\right)_{d_{2}}
$$

)By Proposition 2.5(

$$
=\exp d_{1} X \cdot \exp d_{2} Y \cdot \exp -\frac{1}{2} d_{1} d_{2}[X, Y]
$$

)By Proposition 4.9(,

while we have

$$
\exp d_{1} X+d_{2} Y=\exp d_{2} Y+d_{1} X=\exp d_{2} Y \cdot \exp d_{1} X . \exp -\frac{1}{2} d_{1} d_{2}[Y, X]
$$

by the same argument. Therefore we have

$$
\exp d_{1} X \cdot \exp d_{2} Y \cdot \exp -\frac{1}{2} d_{1} d_{2}[X, Y]=\exp d_{2} Y \cdot \exp d_{1} X . \exp -\frac{1}{2} d_{1} d_{2}[Y, X] \text {. }
$$

By multiplying

$$
\exp \frac{1}{2} d_{1} d_{2}[X, Y]
$$

from the right and making use of Proposition 5.4, we get the desired formula.

Now we deal with the right derivative.

Definition 5.6. Given a microlinear space $M$ and a function $f: M \rightarrow G$, the function

is defined to be such that

$$
\delta^{\text {right }} f: \mathbf{T} M \rightarrow \mathfrak{g}
$$

$$
(\mathbf{d} f(X))_{d}=\left(\delta^{\mathrm{right}} f(X)\right)_{d} \cdot f(x)
$$

for any $x \in M$, any $X \in \mathbf{T}_{x} M$ and any $d \in D$. It is called the right logarithmic derivative of $f$. The restriction of $\delta^{\text {right }} f$ to $\mathbf{T}_{x} M$ is denoted by $\delta^{\text {right }} f(x)$.

Proposition 5.7. Let $M$ be a microlinear space. Given two functions $f, g$ : $M \rightarrow G$ together with $X \in \mathbf{T} M$, we have

$$
\delta^{\text {right }}(f g)(X)=\delta^{\text {right }} f(X)+\operatorname{Ad}(f(x))\left(\delta^{\text {right }} g(X)\right)
$$

with $x=X_{0}$.

Theorem 5.8. Given $X \in \mathfrak{g}$ with $(\operatorname{ad} X)^{n+1}$ vanishing for some natural number $n$, we have

$$
\delta^{\text {right }}(\exp )(X)=\sum_{p=0}^{n} \frac{1}{(p+1) !}(\operatorname{ad} X)^{p} .
$$

6. The Zassenhaus formula

Lemma 6.1. Given $d_{1}, \ldots d_{n} \in D$, we have

$$
\frac{\left(d_{1}+\ldots+d_{n}\right)^{m}}{m !}=\sum_{i_{1}<\ldots<i_{m}} d_{i_{1}} \ldots d_{i_{m}}
$$

for any natural number $m$ with $m \leq n$.

Proof. The reader is referred to Lemma on p. 10 of [8]. 
Theorem 6.2. Given $X, Y \in \mathfrak{g}$ and $d_{1} \in D$, we have

$$
\exp d_{1}(X+Y)=\exp d_{1} X \cdot \exp d_{1} Y \text {. }
$$

Proof. We have

$$
\begin{aligned}
& \exp d_{1}(X+Y)=(X+Y)_{d_{1}} \\
& ) \text { By Proposition } 4.9( \\
= & X_{d_{1}} \cdot Y_{d_{1}} \\
& ) \text { By Proposition } 2.5( \\
= & \exp d_{1} X . \exp d_{1} Y \\
& ) \text { By Proposition } 4.9(.
\end{aligned}
$$

so that we have got to the desired formula.

Theorem 6.3. Given $X, Y \in \mathfrak{g}$ and $d_{1}, d_{2} \in D$, we have

$$
\begin{aligned}
& \exp \left(d_{1}+d_{2}\right)(X+Y) \\
= & \exp \left(d_{1}+d_{2}\right) X \cdot \exp \left(d_{1}+d_{2}\right) Y \cdot \exp -d_{1} d_{2}[X, Y] \\
= & \exp \left(d_{1}+d_{2}\right) X \cdot \exp \left(d_{1}+d_{2}\right) Y \cdot \exp -\frac{\left(d_{1}+d_{2}\right)^{2}}{2}[X, Y]
\end{aligned}
$$

Proof. We have

$$
\begin{aligned}
& \exp \left(d_{1}+d_{2}\right)(X+Y) \\
= & \exp d_{1}(X+Y)+d_{2}(X+Y) \\
= & \exp d_{1}(X+Y) \cdot\left\{\delta^{\text {left }}(\exp )\left(d_{1}(X+Y)\right)(X+Y)\right\}_{d_{2}} \\
& ) \text { left logarithmic derivative }( \\
= & \exp d_{1}(X+Y) \cdot(X+Y)_{d_{2}} \\
& ) \text { By Theorem } 5.3( \\
= & \exp d_{1}(X+Y) \cdot \exp d_{2}(X+Y) \\
& {[\text { By Proposition } 4.9] } \\
= & \exp d_{1} X \cdot \exp d_{1} Y \cdot \exp d_{2} X \cdot \exp d_{2} Y \\
& ) \text { By Theorem } 6.2( \\
= & \exp d_{1} X \cdot \exp d_{2} X \cdot \exp d_{1} Y \cdot \exp d_{1} d_{2}[Y, X] \cdot \exp d_{2} Y \\
& ) \text { By Proposition } 5.5( \\
= & \exp d_{1} X \cdot \exp d_{2} X \cdot \exp d_{1} Y \cdot \exp d_{2} Y \cdot \exp d_{1} d_{2}[Y, X] \\
& ) \text { By Proposition } 5.4( \\
= & \exp \left(d_{1}+d_{2}\right) X \cdot \exp \left(d_{1}+d_{2}\right) Y \cdot \exp d_{1} d_{2}[Y, X] \\
& ) \text { By Proposition } 5.4( \\
= & \exp \left(d_{1}+d_{2}\right) X \cdot \exp \left(d_{1}+d_{2}\right) Y \cdot \exp -d_{1} d_{2}[X, Y]
\end{aligned}
$$

so that we have got to the desired formula. 
Theorem 6.4. Given $X, Y \in \mathfrak{g}$ and $d_{1}, d_{2}, d_{3} \in D$, we have

$$
\begin{aligned}
& \exp \left(d_{1}+d_{2}+d_{3}\right)(X+Y) \\
= & \exp \left(d_{1}+d_{2}+d_{3}\right) X \cdot \exp \left(d_{1}+d_{2}+d_{3}\right) Y \cdot \exp -\left(d_{1} d_{2}+d_{1} d_{3}+d_{2} d_{3}\right)[X, Y] . \\
& \exp d_{1} d_{2} d_{3}[X+2 Y,[X, Y]] \\
= & \exp \left(d_{1}+d_{2}+d_{3}\right) X \cdot \exp \left(d_{1}+d_{2}+d_{3}\right) Y \cdot \exp -\frac{\left(d_{1}+d_{2}+d_{3}\right)^{2}}{2}[X, Y] . \\
& \exp \frac{\left(d_{1}+d_{2}+d_{3}\right)^{3}}{12}[X+2 Y,[X, Y]] .
\end{aligned}
$$

Proof. We have

$$
\exp \left(d_{1}+d_{2}+d_{3}\right)(X+Y)
$$$$
=\exp \left(d_{1}+d_{2}\right)(X+Y)+d_{3}(X+Y)
$$$$
=\exp \left(d_{1}+d_{2}\right)(X+Y) \cdot\left\{\delta^{\text {left }}(\exp )\left(\left(d_{1}+d_{2}\right)(X+Y)\right)(X+Y)\right\}_{d_{3}}
$$

)left logarithmic derivative(

$$
=\exp \left(d_{1}+d_{2}\right)(X+Y) \cdot(X+Y)_{d_{3}}
$$

)By Theorem 5.3(

$$
=\exp \left(d_{1}+d_{2}\right)(X+Y) \cdot \exp d_{3}(X+Y)
$$

)By Proposition 4.9(

$=\exp \left(d_{1}+d_{2}\right) X \cdot \exp \left(d_{1}+d_{2}\right) Y \cdot \exp -d_{1} d_{2}[X, Y] \cdot \exp d_{3} X \cdot \exp d_{3} Y$

)By Theorems 6.2 and 6.3(

$$
\begin{aligned}
& =\exp \left(d_{1}+d_{2}\right) X \cdot \exp d_{1} Y \cdot \exp d_{2} Y \cdot \exp -d_{1} d_{2}[X, Y] \cdot \exp d_{3} X \cdot \exp d_{3} Y \\
& =\exp \left(d_{1}+d_{2}+d_{3}\right) X \cdot \exp d_{1} Y \cdot \exp d_{1} d_{3}[Y, X] \cdot \exp d_{2} Y \cdot \exp d_{2} d_{3}[Y, X] .
\end{aligned}
$$$$
\exp -d_{1} d_{2}[X, Y] \cdot \exp -d_{1} d_{2} d_{3}[[X, Y], X] \cdot \exp d_{3} Y
$$

By moving $\exp d_{3} X$ left towards exp $\left(d_{1}+d_{2}\right) X$
via Propositions 5.4 and 5.5

$=\exp \left(d_{1}+d_{2}+d_{3}\right) X \cdot \exp \left(d_{1}+d_{2}\right) Y \cdot \exp d_{1} d_{3}[Y, X] \cdot \exp d_{1} d_{2} d_{3}[[Y, X], Y]$. $\exp d_{2} d_{3}[Y, X] \cdot \exp -d_{1} d_{2}[X, Y] \cdot \exp -d_{1} d_{2} d_{3}[[X, Y], X] \cdot \exp d_{3} Y$

)By exchanging $\exp d_{1} d_{3}[Y, X]$ and $\exp d_{2} Y$ via Proposition 5.5(

$=\exp \left(d_{1}+d_{2}+d_{3}\right) X \cdot \exp \left(d_{1}+d_{2}+d_{3}\right) Y \cdot \exp d_{1} d_{3}[Y, X]$.

$\exp d_{1} d_{2} d_{3}[[Y, X], Y] \cdot \exp d_{2} d_{3}[Y, X]$.

$\exp -d_{1} d_{2}[X, Y] \cdot \exp -d_{1} d_{2} d_{3}[[X, Y], Y] \cdot \exp -d_{1} d_{2} d_{3}[[X, Y], X]$

By moving $\exp d_{3} Y$ left towards $\exp \left(d_{1}+d_{2}\right) Y$
via Propositions 5.4 and 5.5

$$
=\exp \left(d_{1}+d_{2}+d_{3}\right) X \cdot \exp \left(d_{1}+d_{2}+d_{3}\right) Y \cdot \exp -\left(d_{1} d_{2}+d_{1} d_{3}+d_{2} d_{3}\right)[X, Y] \text {. }
$$

$\exp d_{1} d_{2} d_{3}[X+2 Y,[X, Y]]$

so that we have got to the desired formula. 
Theorem 6.5. Given $X, Y \in \mathfrak{g}$ and $d_{1}, d_{2}, d_{3}, d_{4} \in D$, we have

$$
\begin{aligned}
& \exp \left(d_{1}+d_{2}+d_{3}+d_{4}\right)(X+Y) \\
= & \exp \left(d_{1}+d_{2}+d_{3}+d_{4}\right) X \cdot \exp \left(d_{1}+d_{2}+d_{3}+d_{4}\right) Y . \\
& \exp -\left(d_{1} d_{2}+d_{1} d_{3}+d_{1} d_{4}+d_{2} d_{3}+d_{2} d_{4}+d_{3} d_{4}\right)[X, Y] . \\
& \exp \left(d_{1} d_{2} d_{3}+d_{1} d_{2} d_{4}+d_{1} d_{3} d_{4}+d_{2} d_{3} d_{4}\right)[X+2 Y,[X, Y]] . \\
& \exp d_{1} d_{2} d_{3} d_{4}(-[X,[X,[X, Y]]]-3[X,[Y,[X, Y]]]-3[Y,[Y,[X, Y]]]) \\
= & \exp \left(d_{1}+d_{2}+d_{3}+d_{4}\right) X \cdot \exp \left(d_{1}+d_{2}+d_{3}+d_{4}\right) Y . \\
& \exp -\frac{\left(d_{1}+d_{2}+d_{3}+d_{4}\right)^{2}}{2}[X, Y] . \\
& \exp \frac{\left(d_{1}+d_{2}+d_{3}+d_{4}\right)^{3}}{12}[X+2 Y,[X, Y]] . \\
& \exp \frac{\left(d_{1}+d_{2}+d_{3}+d_{4}\right)^{4}}{24} . \\
& (-[X,[X,[X, Y]]]-3[X,[Y,[X, Y]]]-3[Y,[Y,[X, Y]]]) .
\end{aligned}
$$

Proof. We have

$$
\begin{aligned}
& \exp \left(d_{1}+d_{2}+d_{3}+d_{4}\right)(X+Y) \\
= & \exp \left(d_{1}+d_{2}+d_{3}\right)(X+Y)+d_{4}(X+Y) \\
= & \exp \left(d_{1}+d_{2}+d_{3}\right)(X+Y) . \\
& \left\{\delta^{\text {left }}(\exp )\left(\left(d_{1}+d_{2}+d_{3}\right)(X+Y)\right)((X+Y))\right\}_{d_{4}}
\end{aligned}
$$

)left logarithmic derivative(

$=\exp \left(d_{1}+d_{2}+d_{3}\right)(X+Y) \cdot(X+Y)_{d_{4}}$

)By Theorem 5.3(

$$
=\exp \left(d_{1}+d_{2}+d_{3}\right)(X+Y) \cdot \exp d_{4}(X+Y)
$$

)By Proposition 4.9(

$=\exp \left(d_{1}+d_{2}+d_{3}\right) X \cdot \exp \left(d_{1}+d_{2}+d_{3}\right) Y \cdot \exp -\left(d_{1} d_{2}+d_{1} d_{3}+d_{2} d_{3}\right)[X, Y]$.

$\exp d_{1} d_{2} d_{3}[X+2 Y,[X, Y]] \cdot \exp d_{4} X \cdot \exp d_{4} Y$

)By Theorems 6.2 and 6.4(

$=\exp \left(d_{1}+d_{2}+d_{3}+d_{4}\right) X \cdot \exp d_{1} Y \cdot \exp d_{1} d_{4}[Y, X] \cdot \exp d_{2} Y \cdot \exp d_{2} d_{4}[Y, X]$.

$\exp d_{3} Y \cdot \exp d_{3} d_{4}[Y, X] \cdot \exp -\left(d_{1} d_{2}+d_{1} d_{3}+d_{2} d_{3}\right)[X, Y]$.

$\exp -\left(d_{1} d_{2}+d_{1} d_{3}+d_{2} d_{3}\right) d_{4}[[X, Y], X] \cdot \exp d_{1} d_{2} d_{3}[X+2 Y,[X, Y]]$.

$\exp d_{1} d_{2} d_{3} d_{4}[[X+2 Y,[X, Y]], X] \cdot \exp d_{4} Y$

By moving $\exp d_{4} X$ left towards exp $\left(d_{1}+d_{2}+d_{3}\right) X$
via Propositions 5.4 and 5.5

$=\exp \left(d_{1}+d_{2}+d_{3}+d_{4}\right) X . \exp \left(d_{1}+d_{2}\right) Y$.

$\exp d_{1} d_{4}[Y, X] \cdot \exp d_{1} d_{2} d_{4}[[Y, X], Y]$.

$\exp d_{2} d_{4}[Y, X] \cdot \exp d_{3} Y \cdot \exp d_{3} d_{4}[Y, X] \cdot \exp -\left(d_{1} d_{2}+d_{1} d_{3}+d_{2} d_{3}\right)[X, Y]$.

$\exp -\left(d_{1} d_{2}+d_{1} d_{3}+d_{2} d_{3}\right) d_{4}[[X, Y], X] \cdot \exp d_{1} d_{2} d_{3}[X+2 Y,[X, Y]]$. 
$\exp d_{1} d_{2} d_{3} d_{4}[[X+2 Y,[X, Y]], X] \cdot \exp d_{4} Y$

)By interchanging $\exp d_{1} d_{4}[Y, X]$ and $\exp d_{2} Y$ via Proposition 5.5(

$=\exp \left(d_{1}+d_{2}+d_{3}+d_{4}\right) X \cdot \exp \left(d_{1}+d_{2}+d_{3}\right) Y$.

$\exp d_{1} d_{4}[Y, X] \cdot \exp d_{1} d_{3} d_{4}[[Y, X], Y]$.

$\exp d_{1} d_{2} d_{4}[[Y, X], Y] \cdot \exp d_{1} d_{2} d_{3} d_{4}[[[Y, X], Y], Y] \cdot \exp d_{2} d_{4}[Y, X]$.

$\exp d_{2} d_{3} d_{4}[[Y, X], Y] \cdot \exp d_{3} d_{4}[Y, X] \cdot \exp -\left(d_{1} d_{2}+d_{1} d_{3}+d_{2} d_{3}\right)[X, Y]$.

$\exp -\left(d_{1} d_{2}+d_{1} d_{3}+d_{2} d_{3}\right) d_{4}[[X, Y], X] \cdot \exp d_{1} d_{2} d_{3}[X+2 Y,[X, Y]]$.

$\exp d_{1} d_{2} d_{3} d_{4}[[X+2 Y,[X, Y]], X] \cdot \exp d_{4} Y$

By moving $\exp d_{3} Y$ left towards $\exp \left(d_{1}+d_{2}\right) Y$
via Propositions 5.4 and 5.5

We go on:

$$
\begin{aligned}
&= \exp \left(d_{1}+d_{2}+d_{3}+d_{4}\right) X \cdot \exp \left(d_{1}+d_{2}+d_{3}+d_{4}\right) Y \cdot \exp d_{1} d_{4}[Y, X] . \\
& \exp d_{1} d_{3} d_{4}[[Y, X], Y] \cdot \exp d_{1} d_{2} d_{4}[[Y, X], Y] \cdot \exp d_{1} d_{2} d_{3} d_{4}[[[Y, X], Y], Y] . \\
& \exp d_{2} d_{4}[Y, X] \cdot \exp d_{2} d_{3} d_{4}[[Y, X], Y] \cdot \exp d_{3} d_{4}[Y, X] \cdot \\
& \exp -\left(d_{1} d_{2}+d_{1} d_{3}+d_{2} d_{3}\right)[X, Y] \cdot \exp -\left(d_{1} d_{2}+d_{1} d_{3}+d_{2} d_{3}\right) d_{4}[[X, Y], Y] . \\
& \exp -\left(d_{1} d_{2}+d_{1} d_{3}+d_{2} d_{3}\right) d_{4}[[X, Y], X] \cdot \exp d_{1} d_{2} d_{3}[X+2 Y,[X, Y]] . \\
& \exp d_{1} d_{2} d_{3} d_{4}[[X+2 Y,[X, Y]], Y] \cdot \exp d_{1} d_{2} d_{3} d_{4}[[X+2 Y,[X, Y]], X] \\
& \quad \text { By moving } \exp d_{4} Y \text { left towards } \exp \left(d_{1}+d_{2}+d_{3}\right) Y \\
& \quad \operatorname{via} \text { Propositions } 5.4 \operatorname{and} 5.5 \\
& \exp \left(d_{1}+d_{2}+d_{3}+d_{4}\right) X . \exp \left(d_{1}+d_{2}+d_{3}+d_{4}\right) Y . \\
& \exp -\left(d_{1} d_{2}+d_{1} d_{3}+d_{1} d_{4}+d_{2} d_{3}+d_{2} d_{4}+d_{3} d_{4}\right)[X, Y] . \\
& \exp \left(d_{1} d_{2} d_{3}+d_{1} d_{2} d_{4}+d_{1} d_{3} d_{4}+d_{2} d_{3} d_{4}\right)[X+2 Y,[X, Y]] . \\
& \exp d_{1} d_{2} d_{3} d_{4}(-[X,[X,[X, Y]]]-3[X,[Y,[X, Y]]]-3[Y,[Y,[X, Y]]])
\end{aligned}
$$

so that we have got to the desired formula.

We could go on, but the complexity of computation increases rapidly.

7. The First Approach to The BAKer-CAmpbell-HausdorfF Formula

The following result is no other than Theorem 6.2 itself.

Theorem 7.1. Given $X, Y \in \mathfrak{g}$ and $d_{1} \in D$, we have

$$
\exp d_{1} X . \exp d_{1} Y=\exp d_{1}(X+Y) .
$$

Corollary 7.2. Given $X_{1}, \ldots, X_{n} \in \mathfrak{g}$ and $d_{1} \in D$, we have

$$
\exp d_{1} X_{1} \cdot \exp d_{1} X_{2} \ldots \exp d_{1} X_{n}
$$$$
=\exp d_{1}\left(X_{1}+X_{2}+\ldots+X_{n}\right) \text {. }
$$

Proof. By simple induction on $n$. 
Theorem 7.3. Given $X, Y \in \mathfrak{g}$ and $d_{1}, d_{2} \in D$, we have

$$
\begin{aligned}
& \exp \left(d_{1}+d_{2}\right) X \cdot \exp \left(d_{1}+d_{2}\right) Y \\
= & \exp \left(d_{1}+d_{2}\right)(X+Y)+d_{1} d_{2}[X, Y] \\
= & \exp \left(d_{1}+d_{2}\right)(X+Y)+\frac{\left(d_{1}+d_{2}\right)^{2}}{2}[X, Y] .
\end{aligned}
$$

Proof. We have

$$
\begin{aligned}
& \exp \left(d_{1}+d_{2}\right)(X+Y) \\
= & \exp d_{1}(X+Y)+d_{2}(X+Y) \\
= & \exp d_{1}(X+Y) \cdot\left\{\delta^{\operatorname{left}}(\exp )\left(d_{1}(X+Y)\right)(X+Y)\right\}_{d_{2}} \\
& ) \text { left logarithmic derivative }( \\
= & \exp d_{1}(X+Y) \cdot(X+Y)_{d_{2}} \\
& ) \text { By Theorem } 5.3( \\
= & \exp d_{1}(X+Y) \cdot \exp d_{2}(X+Y) \\
& ) \text { By Proposition } 4.9( \\
= & \exp d_{1} X \cdot \exp d_{1} Y \cdot \exp d_{2} X . \exp d_{2} Y \\
& ) \text { By Theorem } 6.2( \\
= & \exp d_{1} X \cdot \exp d_{2} X \cdot \exp d_{1} Y \cdot \exp d_{1} d_{2}[Y, X] \cdot \exp d_{2} Y \\
& ) \text { By Proposition } 5.5( \\
= & \exp d_{1} X . \exp d_{2} X . \exp d_{1} Y . \exp d_{2} Y \cdot \exp d_{1} d_{2}[Y, X] \\
& ) \text { By Proposition } 5.4( \\
= & \exp \left(d_{1}+d_{2}\right) X . \exp \left(d_{1}+d_{2}\right) Y . \exp d_{1} d_{2}[Y, X] \\
& ) \text { By Proposition } 5.4(
\end{aligned}
$$

so that we get the desired formula by multiplying exp $d_{1} d_{2}[X, Y]$ from the right and making use of Proposition 5.4.

Corollary 7.4 (cf. Theorem 2.12.4 of [17]). Given $X_{1}, \ldots, X_{n} \in \mathfrak{g}$ and $d_{1}, d_{2} \in$ $D$, we have

$$
\begin{aligned}
& \exp \left(d_{1}+d_{2}\right) X_{1} \cdot \exp \left(d_{1}+d_{2}\right) X_{2} \ldots \cdot \exp \left(d_{1}+d_{2}\right) X_{n} \\
= & \exp \left(d_{1}+d_{2}\right)\left(X_{1}+\ldots+X_{n}\right)+d_{1} d_{2} \sum_{1 \leq i<j \leq n}\left[X_{i}, X_{j}\right] \\
= & \exp \left(d_{1}+d_{2}\right)\left(X_{1}+\ldots+X_{n}\right)+\frac{\left(d_{1}+d_{2}\right)^{2}}{2} \sum_{1 \leq i<j \leq n}\left[X_{i}, X_{j}\right] .
\end{aligned}
$$

Proof. Here we deal only with the case of $n=3$, leaving the general treatment by induction on $n$ to the reader. We note in passing that the case of $n=2$ is no other than Theorem 7.3 itself. We have

$$
\begin{aligned}
& \exp \left(d_{1}+d_{2}\right) X_{1} \cdot \exp \left(d_{1}+d_{2}\right) X_{2} \cdot \exp \left(d_{1}+d_{2}\right) X_{3} \\
= & \exp \left(d_{1}+d_{2}\right)\left(X_{1}+X_{2}\right)+\frac{\left(d_{1}+d_{2}\right)^{2}}{2}\left[X_{1}, X_{2}\right] \cdot \exp \left(d_{1}+d_{2}\right) X_{3}
\end{aligned}
$$


)By Theorem 7.3(

$$
\begin{aligned}
= & \exp \left(d_{1}+d_{2}\right)\left\{\left(X_{1}+X_{2}\right)+\frac{d_{1}+d_{2}}{2}\left[X_{1}, X_{2}\right]\right\} \cdot \exp \left(d_{1}+d_{2}\right) X_{3} \\
= & \exp \left(d_{1}+d_{2}\right)\left\{\left(X_{1}+X_{2}+X_{3}\right)+\frac{d_{1}+d_{2}}{2}\left[X_{1}, X_{2}\right]\right\}+ \\
& d_{1} d_{2}\left[\left(X_{1}+X_{2}\right)+\frac{d_{1}+d_{2}}{2}\left[X_{1}, X_{2}\right], X_{3}\right]
\end{aligned}
$$

)By Theorem 7.3(

$$
=\exp \left(d_{1}+d_{2}\right)\left(X_{1}+X_{2}+X_{3}\right)+d_{1} d_{2}\left(\left[X_{1}, X_{2}\right]+\left[X_{1}, X_{3}\right]+\left[X_{2}, X_{3}\right]\right)
$$

so that we are done.

Theorem 7.5. Given $X, Y \in \mathfrak{g}$ and $d_{1}, d_{2}, d_{3} \in D$, we have

$$
\begin{aligned}
& \exp \left(d_{1}+d_{2}+d_{3}\right) X . \exp \left(d_{1}+d_{2}+d_{3}\right) Y \\
= & \exp \left(d_{1}+d_{2}+d_{3}\right)(X+Y)+\left(d_{1} d_{2}+d_{1} d_{3}+d_{2} d_{3}\right)[X, Y]+ \\
& \frac{1}{2} d_{1} d_{2} d_{3}[X-Y,[X, Y]] \\
= & \exp \left(d_{1}+d_{2}+d_{3}\right) X+\left(d_{1}+d_{2}+d_{3}\right) Y+\frac{\left(d_{1}+d_{2}+d_{3}\right)^{2}}{2}[X, Y]+ \\
& \frac{\left(d_{1}+d_{2}+d_{3}\right)^{3}}{12}[X-Y,[X, Y]] .
\end{aligned}
$$

Proof. We have

$$
\begin{aligned}
& \exp \left(d_{1}+d_{2}+d_{3}\right)(X+Y)+\left(d_{1} d_{2}+d_{1} d_{3}+d_{2} d_{3}\right)[X, Y] \\
= & \exp \left\{\left(d_{1}+d_{2}\right)(X+Y)+d_{1} d_{2}[X, Y]\right\}+d_{3}\left\{(X+Y)+\left(d_{1}+d_{2}\right)[X, Y]\right\} \\
= & \exp \left(\left(d_{1}+d_{2}\right)(X+Y)+d_{1} d_{2}[X, Y]\right) . \\
& \left\{\delta^{\text {left }}(\exp )\left(\left(d_{1}+d_{2}\right)(X+Y)+d_{1} d_{2}[X, Y]\right)\left((X+Y)+\left(d_{1}+d_{2}\right)[X, Y]\right)\right\}_{d_{3}} \\
& ) \text { left logarithmic derivative }( \\
= & \exp \left(\left(d_{1}+d_{2}\right)(X+Y)+d_{1} d_{2}[X, Y]\right) . \\
& \left\{(X+Y)+\left(d_{1}+d_{2}\right)[X, Y]-\frac{1}{2} d_{1} d_{2}[X+Y,[X, Y]]\right\}_{d_{3}}
\end{aligned}
$$

)By Theorem 5.3(

$=\exp \left(d_{1}+d_{2}\right) X \cdot \exp \left(d_{1}+d_{2}\right) Y$.

$$
\left\{(X+Y)+\left(d_{1}+d_{2}\right)[X, Y]-\frac{1}{2} d_{1} d_{2}[X+Y,[X, Y]]\right\}_{d_{3}}
$$

[By Theorem 7.3]

$$
\begin{aligned}
= & \exp \left(d_{1}+d_{2}\right) X \cdot \exp \left(d_{1}+d_{2}\right) Y .(X+Y)_{d_{3}} \cdot\left(\left(d_{1}+d_{2}\right)[X, Y]\right)_{d_{3}} . \\
& \left(-\frac{1}{2} d_{1} d_{2}[X+Y,[X, Y]]\right)_{d_{3}}
\end{aligned}
$$

[By Proposition 2.5] 


$$
\begin{aligned}
= & \exp \left(d_{1}+d_{2}\right) X \cdot \exp \left(d_{1}+d_{2}\right) Y \cdot \exp d_{3}(X+Y) \cdot \exp \left(d_{1}+d_{2}\right) d_{3}[X, Y] . \\
& \exp -\frac{1}{2} d_{1} d_{2} d_{3}[X+Y,[X, Y]]
\end{aligned}
$$

[By Proposition 4.9]

$=\exp \left(d_{1}+d_{2}\right) X \cdot \exp d_{1} Y \cdot \exp d_{2} Y \cdot \exp d_{3} X \cdot \exp d_{3} Y \cdot \exp \left(d_{1}+d_{2}\right) d_{3}[X, Y]$.

$\exp -\frac{1}{2} d_{1} d_{2} d_{3}[X+Y,[X, Y]]$

)By Proposition 5.4(

$=\exp \left(d_{1}+d_{2}\right) X \cdot \exp d_{1} Y \cdot \exp d_{3} X \cdot \exp d_{2} Y \cdot \exp d_{2} d_{3}[Y, X] \cdot \exp d_{3} Y$.

$\exp \left(d_{1}+d_{2}\right) d_{3}[X, Y] \cdot \exp -\frac{1}{2} d_{1} d_{2} d_{3}[X+Y,[X, Y]]$

)By Proposition 5.5(

$=\exp \left(d_{1}+d_{2}\right) X \cdot \exp d_{3} X \cdot \exp d_{1} Y \cdot \exp d_{1} d_{3}[Y, X]$.

$\exp d_{2} Y \cdot \exp d_{2} d_{3}[Y, X] \cdot \exp d_{3} Y$.

$\exp \left(d_{1}+d_{2}\right) d_{3}[X, Y] . \exp -\frac{1}{2} d_{1} d_{2} d_{3}[X+Y,[X, Y]]$

)By Proposition 5.5( .

We go on

$=\exp \left(d_{1}+d_{2}\right) X \cdot \exp d_{3} X \cdot \exp d_{1} Y \cdot \exp d_{2} Y$.

$\exp d_{1} d_{3}[Y, X] \cdot \exp d_{1} d_{2} d_{3}[[Y, X], Y]$.

$\exp d_{2} d_{3}[Y, X] \cdot \exp d_{3} Y \cdot \exp \left(d_{1}+d_{2}\right) d_{3}[X, Y] \cdot \exp -\frac{1}{2} d_{1} d_{2} d_{3}[X+Y,[X, Y]]$

)By Proposition 5.5(

$=\exp \left(d_{1}+d_{2}+d_{3}\right) X \cdot \exp \left(d_{1}+d_{2}+d_{3}\right) Y \cdot \exp \frac{1}{2} d_{1} d_{2} d_{3}[Y-X,[X, Y]]$

)By repeated use of Proposition 5.4(

so that we get the desired formula by multiplying

$$
\exp \frac{1}{2} d_{1} d_{2} d_{3}[X-Y,[X, Y]]
$$

from the right and making use of Proposition 5.4.

Theorem 7.6. Given $X, Y \in \mathfrak{g}$ and $d_{1}, d_{2}, d_{3}, d_{4} \in D$, we have

$$
\begin{aligned}
& \exp \left(d_{1}+d_{2}+d_{3}+d_{4}\right) X . \exp \left(d_{1}+d_{2}+d_{3}+d_{4}\right) Y \\
= & \exp \left(d_{1}+d_{2}+d_{3}+d_{4}\right) X+\left(d_{1}+d_{2}+d_{3}+d_{4}\right) Y+ \\
& \left(d_{1} d_{2}+d_{1} d_{3}+d_{1} d_{4}+d_{2} d_{3}+d_{2} d_{4}+d_{3} d_{4}\right)[X, Y]+ \\
& \frac{1}{2}\left(d_{1} d_{2} d_{3}+d_{1} d_{2} d_{4}+d_{1} d_{3} d_{4}+d_{2} d_{3} d_{4}\right)[X-Y,[X, Y]]- \\
& d_{1} d_{2} d_{3} d_{4}\left(\frac{1}{2}[X,[X,[X, Y]]]+\frac{1}{2}[Y,[Y,[X, Y]]]+2[X,[Y,[X, Y]]]\right) \\
= & \exp \left(d_{1}+d_{2}+d_{3}+d_{4}\right) X+\left(d_{1}+d_{2}+d_{3}+d_{4}\right) Y+
\end{aligned}
$$




$$
\begin{aligned}
& \frac{\left(d_{1}+d_{2}+d_{3}+d_{4}\right)^{2}}{2}[X, Y]+ \\
& \frac{\left(d_{1}+d_{2}+d_{3}+d_{4}\right)^{3}}{12}[X-Y,[X, Y]]- \\
& \frac{\left(d_{1}+d_{2}+d_{3}+d_{4}\right)^{4}}{24}\left(\frac{1}{2}[X,[X,[X, Y]]]+\frac{1}{2}[Y,[Y,[X, Y]]]+2[X,[Y,[X, Y]]]\right) .
\end{aligned}
$$

Proof. We have

$$
\begin{aligned}
& \exp \left(d_{1}+d_{2}+d_{3}+d_{4}\right) X+\left(d_{1}+d_{2}+d_{3}+d_{4}\right) Y+ \\
& \left(d_{1} d_{2}+d_{1} d_{3}+d_{1} d_{4}+d_{2} d_{3}+d_{2} d_{4}+d_{3} d_{4}\right)[X, Y]+ \\
& \frac{1}{2}\left(d_{1} d_{2} d_{3}+d_{1} d_{2} d_{4}+d_{1} d_{3} d_{4}+d_{2} d_{3} d_{4}\right)[X-Y,[X, Y]] \\
& =\exp \left\{\begin{array}{c}
\left(d_{1}+d_{2}+d_{3}\right)(X+Y)+\left(d_{1} d_{2}+d_{1} d_{3}+d_{2} d_{3}\right)[X, Y]+ \\
\frac{1}{2} d_{1} d_{2} d_{3}[X-Y,[X, Y]]
\end{array}\right\}+ \\
& \left\{\begin{array}{l}
d_{4}(X+Y)+d_{4}\left(d_{1}+d_{2}+d_{3}\right)[X, Y]+ \\
\frac{1}{2} d_{4}\left(d_{1} d_{2}+d_{1} d_{3}+d_{2} d_{3}\right)[X-Y,[X, Y]]
\end{array}\right\} \\
& =\exp \left\{\begin{array}{c}
\left(d_{1}+d_{2}+d_{3}\right) X+\left(d_{1}+d_{2}+d_{3}\right) Y+\left(d_{1} d_{2}+d_{1} d_{3}+d_{2} d_{3}\right)[X, Y]+ \\
\frac{1}{2} d_{1} d_{2} d_{3}[X-Y,[X, Y]]
\end{array}\right\} . \\
& \left\{\begin{array}{c}
\delta^{\text {left }}(\exp )\left(\begin{array}{c}
\left(d_{1}+d_{2}+d_{3}\right)(X+Y)+\left(d_{1} d_{2}+d_{1} d_{3}+d_{2} d_{3}\right)[X, Y]+ \\
\frac{1}{2} d_{1} d_{2} d_{3}[X-Y,[X, Y]] \\
(X+Y)+\left(d_{1}+d_{2}+d_{3}\right)[X, Y]+ \\
\frac{1}{2}\left(d_{1} d_{2}+d_{1} d_{3}+d_{2} d_{3}\right)[X-Y,[X, Y]]
\end{array}\right)
\end{array}\right\}_{d_{4}}
\end{aligned}
$$

)left logarithmic derivative(

$$
\begin{gathered}
=\exp \left\{\begin{array}{c}
\left.\left(d_{1}+d_{2}+d_{3}\right)(X+Y)+\left(d_{1} d_{2}+d_{1} d_{3}+d_{2} d_{3}\right)[X, Y]+\right\} \\
\frac{1}{2} d_{1} d_{2} d_{3}[X-Y,[X, Y]]
\end{array}\right\} . \\
(X+Y)+\left(d_{1}+d_{2}+d_{3}\right)[X, Y]+ \\
\left\{\begin{array}{c}
\frac{1}{2}\left(d_{1} d_{2}+d_{1} d_{3}+d_{2} d_{3}\right)[X-Y,[X, Y]]- \\
\frac{1}{2}\left(d_{1}+d_{2}+d_{3}\right)^{2}[X+Y,[X, Y]]- \\
\frac{1}{8}\left(d_{1}+d_{2}+d_{3}\right)^{3}[X+Y,[X-Y,[X, Y]]]+ \\
\frac{1}{2}\left(d_{1} d_{2}+d_{1} d_{3}+d_{2} d_{3}\right)[X+Y,[X, Y]]+ \\
\frac{1}{4} d_{1} d_{2} d_{3}[X+Y,[X-Y,[X, Y]]]+ \\
\frac{1}{6}\left(d_{1}+d_{2}+d_{3}\right)^{3}[X+Y,[X+Y,[X, Y]]]
\end{array}\right\}_{d_{4}}
\end{gathered}
$$

)By Theorem 5.3(

$$
\begin{aligned}
= & \exp \left\{\begin{array}{c}
\left.\left(d_{1}+d_{2}+d_{3}\right)(X+Y)+\left(d_{1} d_{2}+d_{1} d_{3}+d_{2} d_{3}\right)[X, Y]+\right\} \\
\frac{1}{2} d_{1} d_{2} d_{3}[X-Y,[X, Y]]
\end{array}\right\} . \\
& \left\{\begin{array}{c}
(X+Y)+\left(d_{1}+d_{2}+d_{3}\right)[X, Y]-\left(d_{1} d_{2}+d_{1} d_{3}+d_{2} d_{3}\right)[Y,[X, Y]]+ \\
\frac{1}{2} d_{1} d_{2} d_{3}[X+Y,[X,[X, Y]]]+\frac{3}{2} d_{1} d_{2} d_{3}[X+Y,[Y,[X, Y]]]
\end{array}\right\}_{d_{4}} \\
= & \exp \left(d_{1}+d_{2}+d_{3}\right) X . \exp \left(d_{1}+d_{2}+d_{3}\right) Y . \\
& \left\{\begin{array}{c}
(X+Y)+\left(d_{1}+d_{2}+d_{3}\right)[X, Y]-\left(d_{1} d_{2}+d_{1} d_{3}+d_{2} d_{3}\right)[Y,[X, Y]]+ \\
\frac{1}{2} d_{1} d_{2} d_{3}[X+Y,[X,[X, Y]]]+\frac{3}{2} d_{1} d_{2} d_{3}[X+Y,[Y,[X, Y]]]
\end{array}\right\}_{d_{4}}
\end{aligned}
$$

)By Theorem 7.5( 


$$
\begin{aligned}
= & \exp \left(d_{1}+d_{2}+d_{3}\right) X \cdot \exp \left(d_{1}+d_{2}+d_{3}\right) Y \cdot(X+Y)_{d_{4}} \cdot \\
& \left(\left(d_{1}+d_{2}+d_{3}\right)[X, Y]\right)_{d_{4}} \cdot \\
& \left(-\left(d_{1} d_{2}+d_{1} d_{3}+d_{2} d_{3}\right)[Y,[X, Y]]\right)_{d_{4}} \cdot \\
& \left(\frac{1}{2} d_{1} d_{2} d_{3}[X+Y,[X,[X, Y]]]+\frac{3}{2} d_{1} d_{2} d_{3}[X+Y,[Y,[X, Y]]]\right)_{d_{4}}
\end{aligned}
$$

)By Proposition 2.5(

$$
=\exp \left(d_{1}+d_{2}+d_{3}\right) X \cdot \exp \left(d_{1}+d_{2}+d_{3}\right) Y \cdot \exp d_{4}(X+Y) \text {. }
$$

$\exp d_{4}\left(d_{1}+d_{2}+d_{3}\right)[X, Y]$.

$\exp -d_{4}\left(d_{1} d_{2}+d_{1} d_{3}+d_{2} d_{3}\right)[Y,[X, Y]]$.

$\exp d_{4}\left(\frac{1}{2} d_{1} d_{2} d_{3}[X+Y,[X,[X, Y]]]+\frac{3}{2} d_{1} d_{2} d_{3}[X+Y,[Y,[X, Y]]]\right)$

)By Proposition 4.9(

$=\exp \left(d_{1}+d_{2}+d_{3}\right) X \cdot \exp d_{1} Y \cdot \exp d_{2} Y \cdot \exp d_{3} Y \cdot \exp d_{4} X \cdot \exp d_{4} Y$.

$\exp d_{4}\left(d_{1}+d_{2}+d_{3}\right)[X, Y] \cdot \exp -d_{4}\left(d_{1} d_{2}+d_{1} d_{3}+d_{2} d_{3}\right)[Y,[X, Y]]$.

$\exp d_{4}\left(\frac{1}{2} d_{1} d_{2} d_{3}[X+Y,[X,[X, Y]]]+\frac{3}{2} d_{1} d_{2} d_{3}[X+Y,[Y,[X, Y]]]\right)$

)By Proposition 5.4( .

We go on

$=\exp \left(d_{1}+d_{2}+d_{3}+d_{4}\right) X \cdot \exp d_{1} Y \cdot \exp d_{1} d_{4}[Y, X]$.

$\exp d_{2} Y \cdot \exp d_{2} d_{4}[Y, X] \cdot \exp d_{3} Y$.

$\exp d_{3} d_{4}[Y, X] \cdot \exp d_{4} Y \cdot \exp d_{4}\left(d_{1}+d_{2}+d_{3}\right)[X, Y]$.

$\exp -d_{4}\left(d_{1} d_{2}+d_{1} d_{3}+d_{2} d_{3}\right)[Y,[X, Y]]$.

$\exp d_{4}\left(\frac{1}{2} d_{1} d_{2} d_{3}[X+Y,[X,[X, Y]]]+\frac{3}{2} d_{1} d_{2} d_{3}[X+Y,[Y,[X, Y]]]\right)$

By moving $\exp d_{4} X$ left towards exp $\left(d_{1}+d_{2}+d_{3}\right) X$
via Propositions 5.4 and 5.5

$=\exp \left(d_{1}+d_{2}+d_{3}+d_{4}\right) X \cdot \exp \left(d_{1}+d_{2}\right) Y \cdot \exp d_{1} d_{4}[Y, X]$.

$\exp d_{1} d_{2} d_{4}[[Y, X], Y]$.

$\exp d_{2} d_{4}[Y, X] \cdot \exp d_{3} Y \cdot \exp d_{3} d_{4}[Y, X] \cdot \exp d_{4} Y \cdot \exp d_{4}\left(d_{1}+d_{2}+d_{3}\right)[X, Y]$.

$\exp -d_{4}\left(d_{1} d_{2}+d_{1} d_{3}+d_{2} d_{3}\right)[Y,[X, Y]]$.

$\exp d_{4}\left(\frac{1}{2} d_{1} d_{2} d_{3}[X+Y,[X,[X, Y]]]+\frac{3}{2} d_{1} d_{2} d_{3}[X+Y,[Y,[X, Y]]]\right)$

By exchanging exp $d_{1} d_{4}[Y, X]$ and $\exp d_{2} Y$ via Proposition 5.5
and using Proposition 5.4

$=\exp \left(d_{1}+d_{2}+d_{3}+d_{4}\right) X \cdot \exp \left(d_{1}+d_{2}+d_{3}\right) Y \cdot \exp d_{1} d_{4}[Y, X]$.

$\exp d_{1} d_{3} d_{4}[[Y, X], Y]$.

$\exp d_{1} d_{2} d_{4}[[Y, X], Y] \cdot \exp d_{1} d_{2} d_{3} d_{4}[[[Y, X], Y], Y] \cdot \exp d_{2} d_{4}[Y, X]$. 
$\exp d_{2} d_{3} d_{4}[[Y, X], Y] \cdot \exp d_{3} d_{4}[Y, X] \cdot \exp d_{4} Y \cdot \exp d_{4}\left(d_{1}+d_{2}+d_{3}\right)[X, Y]$. $\exp -d_{4}\left(d_{1} d_{2}+d_{1} d_{3}+d_{2} d_{3}\right)[Y,[X, Y]]$.

$\exp d_{4}\left(\frac{1}{2} d_{1} d_{2} d_{3}[X+Y,[X,[X, Y]]]+\frac{3}{2} d_{1} d_{2} d_{3}[X+Y,[Y,[X, Y]]]\right)$

By moving $\exp d_{3} Y$ left towards exp $\left(d_{1}+d_{2}\right) Y$
via Propositions 5.4 and 5.5

$=\exp \left(d_{1}+d_{2}+d_{3}+d_{4}\right) X \cdot \exp \left(d_{1}+d_{2}+d_{3}+d_{4}\right) Y$.

$\exp d_{1} d_{2} d_{3} d_{4}\left([[[Y, X], Y], Y]+\frac{1}{2}[X+Y,[X,[X, Y]]]+\frac{3}{2}[X+Y,[Y,[X, Y]]]\right)$

)By moving exp $d_{4} Y$ left towards $\exp \left(d_{1}+d_{2}+d_{3}\right) Y$ via Proposition 5.4(

$=\exp \left(d_{1}+d_{2}+d_{3}+d_{4}\right) X \cdot \exp \left(d_{1}+d_{2}+d_{3}+d_{4}\right) Y$.

$\exp d_{1} d_{2} d_{3} d_{4}\left(\begin{array}{c}\left.\frac{1}{2}[X,[X,[X, Y]]]+\frac{1}{2}[Y,[X,[X, Y]]]+\frac{3}{2}[X,[Y,[X, Y]]]+\right) \\ \frac{1}{2}[Y,[Y,[X, Y]]]\end{array}\right)$

$=\exp \left(d_{1}+d_{2}+d_{3}+d_{4}\right) X \cdot \exp \left(d_{1}+d_{2}+d_{3}+d_{4}\right) Y$.

$\exp d_{1} d_{2} d_{3} d_{4}\left(\frac{1}{2}[X,[X,[X, Y]]]+\frac{1}{2}[Y,[Y,[X, Y]]]+2[X,[Y,[X, Y]]]\right)$

)By Lemma 2.10(

so that we get the desired formula by multiplying

$$
\exp -d_{1} d_{2} d_{3} d_{4}\left(\frac{1}{2}[X,[X,[X, Y]]]+\frac{1}{2}[Y,[Y,[X, Y]]]+2[X,[Y,[X, Y]]]\right)
$$

from the right and making use of Proposition 5.4.

We could go on, but the complexity of computation increases rapidly.

8. The Second approach to the Baker-Campbell-HausdorfF Formula

Theorem 8.1. Given $X, Y \in \mathfrak{g}$ and $d_{1} \in D$, we have

$$
\exp d_{1} X \cdot \exp d_{1} Y=\exp d_{1}(X+Y) \text {. }
$$

Proof. By Proposition 5.4.

Theorem 8.2. Given $X, Y \in \mathfrak{g}$ and $d_{1}, d_{2} \in D$, we have

$$
\begin{aligned}
& \exp \left(d_{1}+d_{2}\right) X \cdot \exp \left(d_{1}+d_{2}\right) Y \\
= & \exp \left(d_{1}+d_{2}\right)(X+Y)+\frac{1}{2}\left(d_{1}+d_{2}\right)^{2}[X, Y] .
\end{aligned}
$$

Proof. We have

$\exp \left(d_{1}+d_{2}\right) X \cdot \exp \left(d_{1}+d_{2}\right) Y=\exp d_{1} X+d_{2} X \cdot \exp d_{1} Y+d_{2} Y$

$=\exp d_{2} X \cdot \exp d_{1} X \cdot \exp d_{1} Y \cdot \exp d_{2} Y$

)By Proposition 5.4(

$=\exp d_{2} X \cdot \exp d_{1}(X+Y) \cdot \exp d_{2} Y$

)By Theorem 8.1( 


$$
\begin{aligned}
= & \exp d_{2} X \cdot \exp d_{1}(X+Y) \cdot \exp d_{2}\left\{Y-\frac{1}{2} d_{1}[X, Y]\right\} \cdot \exp \frac{1}{2} d_{1} d_{2}[X, Y] \\
= & \exp d_{2} X \cdot \exp d_{1}(X+Y)+d_{2} Y \cdot \exp \frac{1}{2} d_{1} d_{2}[X, Y] \\
& ) \text { By Theorem } 5.3 \text { with } \delta^{\text {left }}(\exp )\left(d_{1}(X+Y)\right)(Y)=Y-\frac{1}{2} d_{1}[X, Y]( \\
= & \exp -\frac{1}{2} d_{1} d_{2}[Y, X] \cdot \exp d_{2}\left\{X+\frac{1}{2} d_{1}[Y, X]\right\} \cdot \exp d_{1}(X+Y)+d_{2} Y . \\
& \exp \frac{1}{2} d_{1} d_{2}[X, Y] \\
= & \exp -\frac{1}{2} d_{1} d_{2}[Y, X] \cdot \exp \left(d_{1}+d_{2}\right)(X+Y) \cdot \exp \frac{1}{2} d_{1} d_{2}[X, Y] \\
& ) \text { By Theorem } 5.8 \text { with } \delta^{\text {right }}(\exp )\left(d_{1}(X+Y)+d_{2} X\right)(X)=X+\frac{1}{2} d_{1}[Y, X]( \\
= & \exp \left(d_{1}+d_{2}\right)(X+Y)+d_{1} d_{2}[X, Y] .
\end{aligned}
$$

Theorem 8.3. Given $X, Y \in \mathfrak{g}$ and $d_{1}, d_{2}, d_{3} \in D$, we have

$$
\begin{aligned}
& \exp \left(d_{1}+d_{2}+d_{3}\right) X \cdot \exp \left(d_{1}+d_{2}+d_{3}\right) Y \\
= & \exp \left(d_{1}+d_{2}+d_{3}\right)(X+Y)+\frac{1}{2}\left(d_{1}+d_{2}+d_{3}\right)^{2}[X, Y]+ \\
& \frac{1}{12}\left(d_{1}+d_{2}+d_{3}\right)^{3}[X-Y,[X, Y]] .
\end{aligned}
$$

Proof. We have

$$
\begin{aligned}
& \exp \left(d_{1}+d_{2}+d_{3}\right) X \cdot \exp \left(d_{1}+d_{2}+d_{3}\right) Y \\
= & \exp \left(d_{1}+d_{2}\right) X+d_{3} X \cdot \exp \left(d_{1}+d_{2}\right) Y+d_{3} Y \\
= & \exp d_{3} X \cdot \exp \left(d_{1}+d_{2}\right) X \cdot \exp \left(d_{1}+d_{2}\right) Y \cdot \exp d_{3} Y
\end{aligned}
$$

)By Proposition 5.4(

$=\exp d_{3} X \cdot \exp \left(d_{1}+d_{2}\right)(X+Y)+\frac{1}{2}\left(d_{1}+d_{2}\right)^{2}[X, Y] \cdot \exp d_{3} Y$

)By Theorem 8.2(

$$
\begin{aligned}
= & \exp d_{3} X . \exp \left(d_{1}+d_{2}\right)(X+Y)+\frac{1}{2}\left(d_{1}+d_{2}\right)^{2}[X, Y] . \\
& \exp d_{3}\left\{\begin{array}{c}
Y-\frac{1}{2}\left(\left(d_{1}+d_{2}\right)[X, Y]+\frac{1}{2}\left(d_{1}+d_{2}\right)^{2}[[X, Y], Y]\right)+ \\
\frac{1}{6}\left(d_{1}+d_{2}\right)^{2}[X+Y,[X, Y]]
\end{array}\right\} . \\
& \exp d_{3}\left\{\begin{array}{c}
\frac{1}{2}\left(\left(d_{1}+d_{2}\right)[X, Y]+\frac{1}{2}\left(d_{1}+d_{2}\right)^{2}[[X, Y], Y]\right)- \\
\frac{1}{6}\left(d_{1}+d_{2}\right)^{2}[X+Y,[X, Y]]
\end{array}\right\} \\
= & \exp d_{3} X \cdot \exp \left(d_{1}+d_{2}\right)(X+Y)+\frac{1}{2}\left(d_{1}+d_{2}\right)^{2}[X, Y]+d_{3} Y .
\end{aligned}
$$




$$
\exp d_{3}\left\{\begin{array}{c}
\frac{1}{2}\left(\left(d_{1}+d_{2}\right)[X, Y]+\frac{1}{2}\left(d_{1}+d_{2}\right)^{2}[[X, Y], Y]\right)- \\
\frac{1}{6}\left(d_{1}+d_{2}\right)^{2}[X+Y,[X, Y]]
\end{array}\right\}
$$

$$
\begin{gathered}
\text { By Theorem } 5.3 \text { with } \\
\delta^{\text {left }}(\exp )\left(\left(d_{1}+d_{2}\right)(X+Y)+\frac{1}{2}\left(d_{1}+d_{2}\right)^{2}[X, Y]\right)(Y) \\
=Y-\frac{1}{2}\left(\left(d_{1}+d_{2}\right)[X, Y]+\frac{1}{2}\left(d_{1}+d_{2}\right)^{2}[[X, Y], Y]\right)+ \\
\frac{1}{6}\left(d_{1}+d_{2}\right)^{2}[X+Y,[X, Y]]
\end{gathered} .
$$

We go on

$$
\begin{aligned}
&= \exp d_{3} X \cdot \exp \left(d_{1}+d_{2}\right)(X+Y)+\frac{1}{2}\left(d_{1}+d_{2}\right)^{2}[X, Y]+d_{3} Y . \\
& \exp d_{3}\left\{\frac{1}{2}\left(d_{1}+d_{2}\right)[X, Y]-\frac{1}{4}\left(d_{1}+d_{2}\right)^{2}[X+Y,[X, Y]]\right\} \\
& \exp d_{3}\left\{\begin{array}{c}
\left.\frac{1}{4}\left(d_{1}+d_{2}\right)^{2}[[X, Y], Y]-\frac{1}{6}\left(d_{1}+d_{2}\right)^{2}[X+Y,[X, Y]]+\right\} \\
\frac{1}{4}\left(d_{1}+d_{2}\right)^{2}[X+Y,[X, Y]]
\end{array}\right\} \\
&= \exp d_{3} X . \\
& \exp \left(d_{1}+d_{2}\right)(X+Y)+\frac{1}{2}\left(d_{1}+d_{2}\right)^{2}[X, Y]+d_{3}\left\{Y+\frac{1}{2}\left(d_{1}+d_{2}\right)[X, Y]\right\} \\
& \exp d_{3}\left\{\begin{array}{c}
\frac{1}{4}\left(d_{1}+d_{2}\right)^{2}[[X, Y], Y]-\frac{1}{6}\left(d_{1}+d_{2}\right)^{2}[X+Y,[X, Y]]+ \\
\frac{1}{4}\left(d_{1}+d_{2}\right)^{2}[X+Y,[X, Y]] \\
\text { By Theorem } 5.3 \text { with }
\end{array}\right\} \\
&\left.\quad \begin{array}{c}
\left(\frac{1}{2}\left(d_{1}+d_{2}\right)[X, Y]\right) \\
\delta^{\operatorname{left}}(\exp )\left(\left(d_{1}+d_{2}\right)(X+Y)+\frac{1}{2}\left(d_{1}+d_{2}\right)^{2}[X, Y]+d_{3} Y\right)
\end{array}\right\} . \\
&=\frac{1}{2}\left(d_{1}+d_{2}\right)[X, Y]-\frac{1}{4}\left(d_{1}+d_{2}\right)^{2}[X+Y,[X, Y]]
\end{aligned}
$$

We go on again

$$
\begin{aligned}
= & \exp d_{3}\left\{\begin{array}{c}
-\frac{1}{2}\left(\left(d_{1}+d_{2}\right)[Y, X]+\frac{1}{2}\left(d_{1}+d_{2}\right)^{2}[[X, Y], X]\right)- \\
\frac{1}{6}\left(d_{1}+d_{2}\right)^{2}[X+Y,[Y, X]]
\end{array}\right\} . \\
& \exp d_{3}\left\{\begin{array}{c}
X+\frac{1}{2}\left(\left(d_{1}+d_{2}\right)[Y, X]+\frac{1}{2}\left(d_{1}+d_{2}\right)^{2}[[X, Y], X]\right)+ \\
\frac{1}{6}\left(d_{1}+d_{2}\right)^{2}[X+Y,[Y, X]]
\end{array}\right\} . \\
& \exp \left(d_{1}+d_{2}\right)(X+Y)+\frac{1}{2}\left(d_{1}+d_{2}\right)^{2}[X, Y]+d_{3}\left\{Y+\frac{1}{2}\left(d_{1}+d_{2}\right)[X, Y]\right\} . \\
& \exp d_{3}\left\{\begin{array}{c}
\frac{1}{4}\left(d_{1}+d_{2}\right)^{2}[[X, Y], Y]-\frac{1}{6}\left(d_{1}+d_{2}\right)^{2}[X+Y,[X, Y]]+ \\
\frac{1}{4}\left(d_{1}+d_{2}\right)^{2}[X+Y,[X, Y]]
\end{array}\right\} \\
= & \exp d_{3}\left\{\begin{array}{c}
-\frac{1}{2}\left(\left(d_{1}+d_{2}\right)[Y, X]+\frac{1}{2}\left(d_{1}+d_{2}\right)^{2}[[X, Y], X]\right)- \\
\frac{1}{6}\left(d_{1}+d_{2}\right)^{2}[X+Y,[Y, X]]
\end{array}\right\} . \\
& \exp \left(d_{1}+d_{2}\right)(X+Y)+\frac{1}{2}\left(d_{1}+d_{2}\right)^{2}[X, Y]+ \\
& d_{3}\left\{(X+Y)+\frac{1}{2}\left(d_{1}+d_{2}\right)[X, Y]\right\} .
\end{aligned}
$$




$$
\begin{aligned}
& \exp d_{3}\left\{\begin{array}{c}
\frac{1}{4}\left(d_{1}+d_{2}\right)^{2}[[X, Y], Y]-\frac{1}{6}\left(d_{1}+d_{2}\right)^{2}[X+Y,[X, Y]]+ \\
\frac{1}{4}\left(d_{1}+d_{2}\right)^{2}[X+Y,[X, Y]]
\end{array}\right\} \\
& \begin{array}{c}
\text { By Theorem } 5.8 \text { with } \\
\delta^{\text {right }}(\exp )\left(\begin{array}{c}
\left(d_{1}+d_{2}\right)(X+Y)+\frac{1}{2}\left(d_{1}+d_{2}\right)^{2}[X, Y]+ \\
d_{3}\left\{Y+\frac{1}{2}\left(d_{1}+d_{2}\right)[X, Y]\right\}
\end{array}\right)(X) \\
=X+\frac{1}{2}\left(\left(d_{1}+d_{2}\right)[Y, X]+\frac{1}{2}\left(d_{1}+d_{2}\right)^{2}[[X, Y], X]\right)+ \\
\frac{1}{6}\left(d_{1}+d_{2}\right)^{2}[X+Y,[Y, X]]
\end{array} \\
& =\exp d_{3}\left\{\begin{array}{c}
-\frac{1}{4}\left(d_{1}+d_{2}\right)^{2}[[X, Y], X]-\frac{1}{6}\left(d_{1}+d_{2}\right)^{2}[X+Y,[Y, X]]+ \\
\frac{1}{4}\left(d_{1}+d_{2}\right)^{2}[X+Y,[Y, X]]
\end{array}\right\} . \\
& \exp d_{3}\left\{-\frac{1}{2}\left(d_{1}+d_{2}\right)[Y, X]-\frac{1}{4}\left(d_{1}+d_{2}\right)^{2}[X+Y,[Y, X]]\right\} \text {. } \\
& \exp \left(d_{1}+d_{2}\right)(X+Y)+\frac{1}{2}\left(d_{1}+d_{2}\right)^{2}[X, Y]+ \\
& d_{3}\left\{(X+Y)+\frac{1}{2}\left(d_{1}+d_{2}\right)[X, Y]\right\} \text {. } \\
& \exp d_{3}\left\{\begin{array}{c}
\frac{1}{4}\left(d_{1}+d_{2}\right)^{2}[[X, Y], Y]-\frac{1}{6}\left(d_{1}+d_{2}\right)^{2}[X+Y,[X, Y]]+ \\
\frac{1}{4}\left(d_{1}+d_{2}\right)^{2}[X+Y,[X, Y]]
\end{array}\right\} \\
& =\exp d_{3}\left\{\begin{array}{c}
-\frac{1}{4}\left(d_{1}+d_{2}\right)^{2}[[X, Y], X]-\frac{1}{6}\left(d_{1}+d_{2}\right)^{2}[X+Y,[Y, X]]+ \\
\frac{1}{4}\left(d_{1}+d_{2}\right)^{2}[X+Y,[Y, X]]
\end{array}\right\} . \\
& \exp \left(d_{1}+d_{2}+d_{3}\right)(X+Y)+\frac{1}{2}\left(d_{1}+d_{2}+d_{3}\right)^{2}[X, Y] \text {. } \\
& \exp d_{3}\left\{\begin{array}{c}
\frac{1}{4}\left(d_{1}+d_{2}\right)^{2}[[X, Y], Y]-\frac{1}{6}\left(d_{1}+d_{2}\right)^{2}[X+Y,[X, Y]]+ \\
\frac{1}{4}\left(d_{1}+d_{2}\right)^{2}[X+Y,[X, Y]]
\end{array}\right\}
\end{aligned}
$$

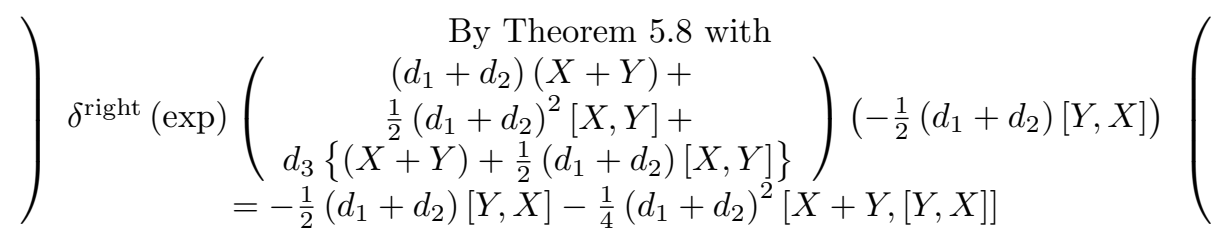$$
=\exp \left(d_{1}+d_{2}+d_{3}\right)(X+Y)+\frac{1}{2}\left(d_{1}+d_{2}+d_{3}\right)^{2}[X, Y]+
$$$$
\frac{1}{4}\left(d_{1}+d_{2}\right)^{2} d_{3}([X,[X, Y]]-[Y,[X, Y]])
$$$$
=\exp \left(d_{1}+d_{2}+d_{3}\right)(X+Y)+\frac{1}{2}\left(d_{1}+d_{2}+d_{3}\right)^{2}[X, Y]+
$$$$
\frac{1}{12}\left(d_{1}+d_{2}+d_{3}\right)^{3}[X-Y,[X, Y]] \text {. }
$$

Theorem 8.4. Given $X, Y \in \mathfrak{g}$ and $d_{1}, d_{2}, d_{3}, d_{4} \in D$, we have

$$
\begin{aligned}
& \exp \left(d_{1}+d_{2}+d_{3}+d_{4}\right) X \cdot \exp \left(d_{1}+d_{2}+d_{3}+d_{4}\right) Y \\
= & \exp \left(d_{1}+d_{2}+d_{3}+d_{4}\right)(X+Y)+\frac{\left(d_{1}+d_{2}+d_{3}+d_{4}\right)^{2}}{2}[X, Y]+
\end{aligned}
$$




$$
\begin{aligned}
& \frac{\left(d_{1}+d_{2}+d_{3}+d_{4}\right)^{3}}{12}[X-Y,[X, Y]]- \\
& \frac{\left(d_{1}+d_{2}+d_{3}+d_{4}\right)^{4}}{48}\left(\begin{array}{c}
{[X,[Y,[X, Y]]]+[Y,[X,[X, Y]]]+} \\
{[X+Y,[X+Y,[X, Y]]]}
\end{array}\right) .
\end{aligned}
$$

Proof. We have

$$
\begin{aligned}
& \exp \left(d_{1}+d_{2}+d_{3}+d_{4}\right) X \cdot \exp \left(d_{1}+d_{2}+d_{3}+d_{4}\right) Y \\
= & \exp \left(d_{1}+d_{2}+d_{3}\right) X+d_{4} X \cdot \exp \left(d_{1}+d_{2}+d_{3}\right) Y+d_{4} Y \\
= & \exp d_{4} X \cdot \exp \left(d_{1}+d_{2}+d_{3}\right) X \cdot \exp \left(d_{1}+d_{2}+d_{3}\right) Y \cdot \exp d_{4} Y
\end{aligned}
$$

)By Proposition 5.4(

$$
\begin{aligned}
= & \exp d_{4} X . \exp \left(d_{1}+d_{2}+d_{3}\right)(X+Y)+\frac{1}{2}\left(d_{1}+d_{2}+d_{3}\right)^{2}[X, Y]+ \\
& \frac{1}{12}\left(d_{1}+d_{2}+d_{3}\right)^{3}[X-Y,[X, Y]] . \exp d_{4} Y
\end{aligned}
$$

)By Theorem 8.3(

$$
\begin{aligned}
& =\exp d_{4} X . \exp \left(d_{1}+d_{2}+d_{3}\right)(X+Y)+\frac{1}{2}\left(d_{1}+d_{2}+d_{3}\right)^{2}[X, Y]+ \\
& \frac{1}{12}\left(d_{1}+d_{2}+d_{3}\right)^{3}[X-Y,[X, Y]] \text {. } \\
& \exp d_{4}\left\{\begin{array}{c}
Y-\frac{1}{2}\left(\begin{array}{c}
\left(d_{1}+d_{2}+d_{3}\right)[X, Y]+\frac{1}{2}\left(d_{1}+d_{2}+d_{3}\right)^{2}[[X, Y], Y]+ \\
\frac{1}{12}\left(d_{1}+d_{2}+d_{3}\right)^{3}[[X-Y,[X, Y]], Y] \\
\left(d_{1}+d_{2}+d_{3}\right)^{2}[X+Y,[X, Y]]+ \\
\frac{1}{2}\left(d_{1}+d_{2}+d_{3}\right)^{3}[X+Y,[[X, Y], Y]]
\end{array}\right)+
\end{array}\right\} .
\end{aligned}
$$

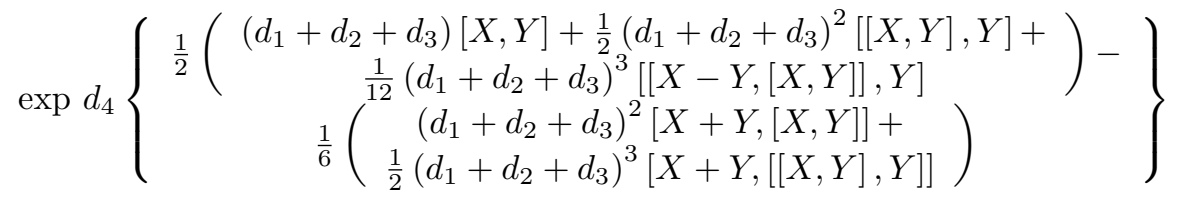

$$
\begin{aligned}
& =\exp d_{4} X . \exp \left(d_{1}+d_{2}+d_{3}\right)(X+Y)+\frac{1}{2}\left(d_{1}+d_{2}+d_{3}\right)^{2}[X, Y]+
\end{aligned}
$$$$
\frac{1}{12}\left(d_{1}+d_{2}+d_{3}\right)^{3}[X-Y,[X, Y]]+d_{4} Y \text {. }
$$

$$
\begin{gathered}
\exp d_{4}\left\{\begin{array}{c}
\frac{1}{2}\left(\begin{array}{c}
\left(d_{1}+d_{2}+d_{3}\right)[X, Y]+\frac{1}{2}\left(d_{1}+d_{2}+d_{3}\right)^{2}[[X, Y], Y]+ \\
\frac{1}{12}\left(d_{1}+d_{2}+d_{3}\right)^{3}[[X-Y,[X, Y]], Y] \\
\left(d_{1}+d_{2}+d_{3}\right)^{2}[X+Y,[X, Y]]+ \\
\frac{1}{2}\left(d_{1}+d_{2}+d_{3}\right)^{3}[X+Y,[[X, Y], Y]]
\end{array}\right)- \\
\text { By Theorem } 5.3 \text { with }
\end{array}\right\} \\
\delta^{\operatorname{left}}(\exp )\left(\begin{array}{c}
\left(d_{1}+d_{2}+d_{3}\right)(X+Y)+\frac{1}{2}\left(d_{1}+d_{2}+d_{3}\right)^{2}[X, Y]+ \\
\frac{1}{12}\left(d_{1}+d_{2}+d_{3}\right)^{3}[X-Y,[X, Y]]
\end{array}\right)(Y) \\
=Y-\frac{1}{2}\left(\begin{array}{c}
\left(d_{1}+d_{2}+d_{3}\right)[X, Y]+\frac{1}{2}\left(d_{1}+d_{2}+d_{3}\right)^{2}[[X, Y], Y]+ \\
\frac{1}{12}\left(d_{1}+d_{2}+d_{3}\right)^{3}[[X-Y,[X, Y]], Y] \\
\left(d_{1}+d_{2}+d_{3}\right)^{2}[X+Y,[X, Y]]+ \\
\frac{1}{2}\left(d_{1}+d_{2}+d_{3}\right)^{3}[X+Y,[[X, Y], Y]]
\end{array}\right)+
\end{gathered}
$$


We go on

$$
\begin{aligned}
& =\exp d_{4} X . \exp \left(d_{1}+d_{2}+d_{3}\right)(X+Y)+\frac{1}{2}\left(d_{1}+d_{2}+d_{3}\right)^{2}[X, Y]+ \\
& \frac{1}{12}\left(d_{1}+d_{2}+d_{3}\right)^{3}[X-Y,[X, Y]]+d_{4} Y \text {. } \\
& \exp d_{4}\left\{\begin{array}{c}
\frac{1}{2}\left(d_{1}+d_{2}+d_{3}\right)[X, Y]+\frac{1}{4}\left(d_{1}+d_{2}+d_{3}\right)^{2}[[X, Y], Y]- \\
\frac{1}{6}\left(d_{1}+d_{2}+d_{3}\right)^{2}[X+Y,[X, Y]]- \\
\frac{1}{2}\left(d_{1}+d_{2}+d_{3}\right)^{2}[X+Y,[X, Y]]+ \\
\frac{1}{4}\left(d_{1}+d_{2}+d_{3}\right)^{3}[X+Y,[[X, Y], Y]]- \\
\frac{1}{6}\left(d_{1}+d_{2}+d_{3}\right)^{3}[X+Y,[X+Y,[X, Y]]] \\
\frac{1}{12}\left(d_{1}+d_{2}+d_{3}\right)^{3}[X+Y,[X+Y,[X, Y]]]
\end{array}\right\} . \\
& \exp d_{4}\left\{\begin{array}{c}
\frac{1}{24}\left(d_{1}+d_{2}+d_{3}\right)^{3}[[X-Y,[X, Y]], Y]- \\
\frac{1}{12}\left(d_{1}+d_{2}+d_{3}\right)^{3}[X+Y,[[X, Y], Y]]+ \\
\frac{1}{2}\left(d_{1}+d_{2}+d_{3}\right)^{2}[X+Y,[X, Y]]+ \\
\frac{1}{4}\left(d_{1}+d_{2}+d_{3}\right)^{3}[X+Y,[[X, Y], Y]]- \\
\frac{1}{6}\left(d_{1}+d_{2}+d_{3}\right)^{3}[X+Y,[X+Y,[X, Y]]] \\
\frac{1}{12}\left(d_{1}+d_{2}+d_{3}\right)^{3}[X+Y,[X+Y,[X, Y]]]
\end{array}\right\} \\
& =\exp d_{4} X \cdot \exp \left(d_{1}+d_{2}+d_{3}\right)(X+Y)+\frac{1}{2}\left(d_{1}+d_{2}+d_{3}\right)^{2}[X, Y]+ \\
& \frac{1}{12}\left(d_{1}+d_{2}+d_{3}\right)^{3}[X-Y,[X, Y]]+ \\
& d_{4}\left\{\begin{array}{c}
Y+\frac{1}{2}\left(d_{1}+d_{2}+d_{3}\right)[X, Y]+\frac{1}{4}\left(d_{1}+d_{2}+d_{3}\right)^{2}[[X, Y], Y]- \\
\frac{1}{6}\left(d_{1}+d_{2}+d_{3}\right)^{2}[X+Y,[X, Y]]
\end{array}\right\} . \\
& \exp d_{4}\left\{\begin{array}{c}
\frac{1}{24}\left(d_{1}+d_{2}+d_{3}\right)^{3}[[X-Y,[X, Y]], Y]- \\
\frac{1}{12}\left(d_{1}+d_{2}+d_{3}\right)^{3}[X+Y,[[X, Y], Y]]+ \\
\frac{1}{2}\left(d_{1}+d_{2}+d_{3}\right)^{2}[X+Y,[X, Y]]+ \\
\frac{1}{4}\left(d_{1}+d_{2}+d_{3}\right)^{3}[X+Y,[[X, Y], Y]]- \\
\frac{1}{6}\left(d_{1}+d_{2}+d_{3}\right)^{3}[X+Y,[X+Y,[X, Y]]] \\
\frac{1}{12}\left(d_{1}+d_{2}+d_{3}\right)^{3}[X+Y,[X+Y,[X, Y]]]
\end{array}\right\} \\
& \left.\begin{array}{c}
\text { By Theorem 5.3 with } \\
\left(d_{1}+d_{2}+d_{3}\right)(X+Y)+ \\
\frac{1}{2}\left(d_{1}+d_{2}+d_{3}\right)^{2}[X, Y]+ \\
\frac{1}{12}\left(d_{1}+d_{2}+d_{3}\right)^{3}[X-Y,[X, Y]]+ \\
d_{4} Y \\
\frac{1}{2}\left(d_{1}+d_{2}+d_{3}\right)[X, Y]+ \\
\delta^{\text {left }}(\exp )\left(\begin{array}{c}
1 \\
\frac{1}{4}\left(d_{1}+d_{2}+d_{3}\right)^{2}[[X, Y], Y]- \\
\frac{1}{6}\left(d_{1}+d_{2}+d_{3}\right)^{2}[X+Y,[X, Y]]
\end{array}\right) \\
=\frac{1}{2}\left(d_{1}+d_{2}+d_{3}\right)[X, Y]+\frac{1}{4}\left(d_{1}+d_{2}+d_{3}\right)^{2}[[X, Y], Y]- \\
\frac{1}{6}\left(d_{1}+d_{2}+d_{3}\right)^{2}[X+Y,[X, Y]]- \\
\frac{1}{2}\left(d_{1}+d_{2}+d_{3}\right)^{2}[X+Y,[X, Y]]+ \\
\frac{1}{4}\left(d_{1}+d_{2}+d_{3}\right)^{3}[X+Y,[[X, Y], Y]]- \\
\frac{1}{6}\left(d_{1}+d_{2}+d_{3}\right)^{3}[X+Y,[X+Y,[X, Y]]] \\
\frac{1}{12}\left(d_{1}+d_{2}+d_{3}\right)^{3}[X+Y,[X+Y,[X, Y]]]
\end{array}\right)+
\end{aligned}
$$


We go on again

$$
\begin{aligned}
& =\exp d_{4} X \cdot \exp \left(d_{1}+d_{2}+d_{3}\right)(X+Y)+\frac{1}{2}\left(d_{1}+d_{2}+d_{3}\right)^{2}[X, Y]+ \\
& \frac{1}{12}\left(d_{1}+d_{2}+d_{3}\right)^{3}[X-Y,[X, Y]]+ \\
& d_{4}\left\{\begin{array}{c}
Y+\frac{1}{2}\left(d_{1}+d_{2}+d_{3}\right)[X, Y]+\frac{1}{4}\left(d_{1}+d_{2}+d_{3}\right)^{2}[[X, Y], Y]- \\
\frac{1}{6}\left(d_{1}+d_{2}+d_{3}\right)^{2}[X+Y,[X, Y]]
\end{array}\right\} . \\
& \exp d_{4}\left\{\begin{array}{c}
\frac{1}{4}\left(d_{1}+d_{2}+d_{3}\right)^{2}[X+Y,[X, Y]]- \\
\frac{1}{8}\left(d_{1}+d_{2}+d_{3}\right)^{3}[X+Y,[X+Y,[X, Y]]]
\end{array}\right\} . \\
& \exp d_{4}\left\{\begin{array}{c}
\frac{1}{24}\left(d_{1}+d_{2}+d_{3}\right)^{3}[[X-Y,[X, Y]], Y]- \\
\frac{1}{12}\left(d_{1}+d_{2}+d_{3}\right)^{3}[X+Y,[[X, Y], Y]]+ \\
\frac{1}{4}\left(d_{1}+d_{2}+d_{3}\right)^{3}[X+Y,[[X, Y], Y]]- \\
\frac{1}{6}\left(d_{1}+d_{2}+d_{3}\right)^{3}[X+Y,[X+Y,[X, Y]]]- \\
\frac{1}{12}\left(d_{1}+d_{2}+d_{3}\right)^{3}[X+Y,[X+Y,[X, Y]]]+ \\
\frac{1}{8}\left(d_{1}+d_{2}+d_{3}\right)^{3}[X+Y,[X+Y,[X, Y]]]
\end{array}\right\} \\
& =\exp d_{4} X \cdot \exp \left(d_{1}+d_{2}+d_{3}\right)(X+Y)+\frac{1}{2}\left(d_{1}+d_{2}+d_{3}\right)^{2}[X, Y]+
\end{aligned}
$$$$
\frac{1}{12}\left(d_{1}+d_{2}+d_{3}\right)^{3}[X-Y,[X, Y]]+
$$$$
d_{4}\left\{\begin{array}{c}
Y+\frac{1}{2}\left(d_{1}+d_{2}+d_{3}\right)[X, Y]+\frac{1}{4}\left(d_{1}+d_{2}+d_{3}\right)^{2}[[X, Y], Y]- \\
\frac{1}{6}\left(d_{1}+d_{2}+d_{3}\right)^{2}[X+Y,[X, Y]]+\frac{1}{4}\left(d_{1}+d_{2}+d_{3}\right)^{2}[X+Y,[X, Y]]
\end{array}\right\} .
$$$$
\exp d_{4}\left\{\begin{array}{c}
\frac{1}{24}\left(d_{1}+d_{2}+d_{3}\right)^{3}[[X-Y,[X, Y]], Y]- \\
\frac{1}{12}\left(d_{1}+d_{2}+d_{3}\right)^{3}[X+Y,[[X, Y], Y]]+ \\
\frac{1}{4}\left(d_{1}+d_{2}+d_{3}\right)^{3}[X+Y,[[X, Y], Y]]- \\
\frac{1}{6}\left(d_{1}+d_{2}+d_{3}\right)^{3}[X+Y,[X+Y,[X, Y]]]- \\
\frac{1}{12}\left(d_{1}+d_{2}+d_{3}\right)^{3}[X+Y,[X+Y,[X, Y]]]+ \\
\frac{1}{8}\left(d_{1}+d_{2}+d_{3}\right)^{3}[X+Y,[X+Y,[X, Y]]]
\end{array}\right\}
$$$$
\delta^{\text {left }}(\exp )\left(\begin{array}{c}
\text { By Theorem } 5.3 \text { with } \\
\left(d_{1}+d_{2}+d_{3}\right)(X+Y)+ \\
\frac{1}{2}\left(d_{1}+d_{2}+d_{3}\right)^{2}[X, Y]+ \\
\frac{1}{12}\left(d_{1}+d_{2}+d_{3}\right)^{3}[X-Y,[X, Y]]+ \\
d_{4}\left\{\begin{array}{c}
Y+\frac{1}{2}\left(d_{1}+d_{2}+d_{3}\right)[X, Y]+ \\
\frac{1}{4}\left(d_{1}+d_{2}+d_{3}\right)^{2}[[X, Y], Y]- \\
\frac{1}{6}\left(d_{1}+d_{2}+d_{3}\right)^{2}[X+Y,[X, Y]]
\end{array}\right\} \\
\left(\frac{1}{4}\left(d_{1}+d_{2}+d_{3}\right)^{2}[X+Y,[X, Y]]\right) \\
=\frac{1}{4}\left(d_{1}+d_{2}+d_{3}\right)^{2}[X+Y,[X, Y]]- \\
\frac{1}{8}\left(d_{1}+d_{2}+d_{3}\right)^{3}[X+Y,[X+Y,[X, Y]]]
\end{array}\right)
$$

We go on once more

$$
\begin{aligned}
& =\exp d_{4}\left\{\begin{array}{c}
-\frac{1}{2}\left(\begin{array}{c}
\left.\left(d_{1}+d_{2}+d_{3}\right)[Y, X]+\frac{1}{2}\left(d_{1}+d_{2}+d_{3}\right)^{2}[[X, Y], X]+\right)- \\
\frac{1}{12}\left(d_{1}+d_{2}+d_{3}\right)^{3}[[X-Y,[X, Y]], X] \\
\left(d_{1}+d_{2}+d_{3}\right)^{2}[X+Y,[Y, X]]+ \\
\frac{1}{2}\left(d_{1}+d_{2}+d_{3}\right)^{3}[X+Y,[[X, Y], X]]
\end{array}\right)
\end{array}\right\} . \\
& \exp d_{4}\left\{\begin{array}{c}
X+\frac{1}{2}\left(\begin{array}{c}
\left(d_{1}+d_{2}+d_{3}\right)[Y, X]+\frac{1}{2}\left(d_{1}+d_{2}+d_{3}\right)^{2}[[X, Y], X]+ \\
\frac{1}{12}\left(d_{1}+d_{2}+d_{3}\right)^{3}[[X-Y,[X, Y]], X] \\
\left(d_{1}+d_{2}+d_{3}\right)^{2}[X+Y,[Y, X]]+ \\
\frac{1}{2}\left(d_{1}+d_{2}+d_{3}\right)^{3}[X+Y,[[X, Y], X]]
\end{array}\right)+ \\
\frac{1}{6}[X]
\end{array}\right\}
\end{aligned}
$$




$$
\begin{aligned}
& \exp \left(d_{1}+d_{2}+d_{3}\right)(X+Y)+\frac{1}{2}\left(d_{1}+d_{2}+d_{3}\right)^{2}[X, Y]+ \\
& \frac{1}{12}\left(d_{1}+d_{2}+d_{3}\right)^{3}[X-Y,[X, Y]]+ \\
& d_{4}\left\{\begin{array}{c}
Y+\frac{1}{2}\left(d_{1}+d_{2}+d_{3}\right)[X, Y]+\frac{1}{4}\left(d_{1}+d_{2}+d_{3}\right)^{2}[[X, Y], Y]- \\
\frac{1}{6}\left(d_{1}+d_{2}+d_{3}\right)^{2}[X+Y,[X, Y]]+\frac{1}{4}\left(d_{1}+d_{2}+d_{3}\right)^{2}[X+Y,[X, Y]]
\end{array}\right\} . \\
& \exp d_{4}\left\{\begin{array}{c}
\frac{1}{24}\left(d_{1}+d_{2}+d_{3}\right)^{3}[[X-Y,[X, Y]], Y]- \\
\frac{1}{12}\left(d_{1}+d_{2}+d_{3}\right)^{3}[X+Y,[[X, Y], Y]]+ \\
\frac{1}{4}\left(d_{1}+d_{2}+d_{3}\right)^{3}[X+Y,[[X, Y], Y]]- \\
\left.\frac{1}{6}\left(d_{1}+d_{2}+d_{3}\right)^{3}[X+Y,[X+Y,[X, Y]]]\right)- \\
\frac{1}{12}\left(d_{1}+d_{2}+d_{3}\right)^{3}[X+Y,[X+Y,[X, Y]]]+ \\
\frac{1}{8}\left(d_{1}+d_{2}+d_{3}\right)^{3}[X+Y,[X+Y,[X, Y]]]
\end{array}\right\} \\
& =\exp d_{4}\left\{\begin{array}{c}
-\frac{1}{2}\left(\begin{array}{c}
\left.\left(d_{1}+d_{2}+d_{3}\right)[Y, X]+\frac{1}{2}\left(d_{1}+d_{2}+d_{3}\right)^{2}[[X, Y], X]+\right)- \\
\frac{1}{12}\left(d_{1}+d_{2}+d_{3}\right)^{3}[[X-Y,[X, Y]], X] \\
\left(d_{1}+d_{2}+d_{3}\right)^{2}[X+Y,[Y, X]]+ \\
\frac{1}{2}\left(d_{1}+d_{2}+d_{3}\right)^{3}[X+Y,[[X, Y], X]]
\end{array}\right)
\end{array}\right\} . \\
& \exp \left(d_{1}+d_{2}+d_{3}\right)(X+Y)+\frac{1}{2}\left(d_{1}+d_{2}+d_{3}\right)^{2}[X, Y]+ \\
& \frac{1}{12}\left(d_{1}+d_{2}+d_{3}\right)^{3}[X-Y,[X, Y]]+ \\
& d_{4}\left\{\begin{array}{c}
X+Y+\frac{1}{2}\left(d_{1}+d_{2}+d_{3}\right)[X, Y]+\frac{1}{4}\left(d_{1}+d_{2}+d_{3}\right)^{2}[[X, Y], Y]- \\
\frac{1}{6}\left(d_{1}+d_{2}+d_{3}\right)^{2}[X+Y,[X, Y]]+ \\
\frac{1}{4}\left(d_{1}+d_{2}+d_{3}\right)^{2}[X+Y,[X, Y]]
\end{array}\right\} . \\
& \exp d_{4}\left\{\begin{array}{c}
\frac{1}{24}\left(d_{1}+d_{2}+d_{3}\right)^{3}[[X-Y,[X, Y]], Y]- \\
\frac{1}{12}\left(d_{1}+d_{2}+d_{3}\right)^{3}[X+Y,[[X, Y], Y]]+ \\
\frac{1}{4}\left(d_{1}+d_{2}+d_{3}\right)^{3}[X+Y,[[X, Y], Y]]- \\
\left.\frac{1}{6}\left(d_{1}+d_{2}+d_{3}\right)^{3}[X+Y,[X+Y,[X, Y]]]-\right) \\
\frac{1}{12}\left(d_{1}+d_{2}+d_{3}\right)^{3}[X+Y,[X+Y,[X, Y]]]+ \\
\frac{1}{8}\left(d_{1}+d_{2}+d_{3}\right)^{3}[X+Y,[X+Y,[X, Y]]]
\end{array}\right\}
\end{aligned}
$$

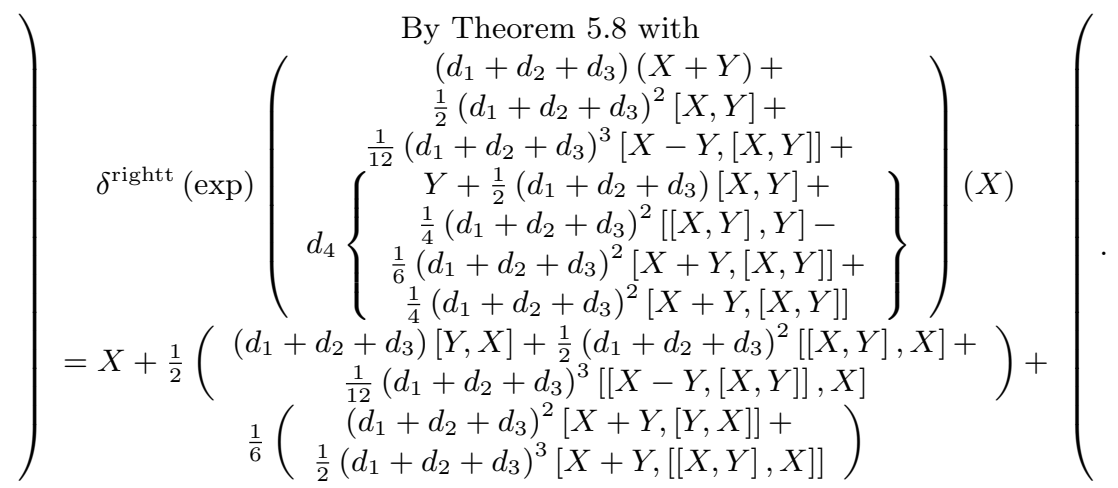

By Theorem 5.8 with 
We go on once more

$$
\begin{gathered}
=\exp d_{4}\left\{\begin{array}{c}
-\frac{1}{24}\left(d_{1}+d_{2}+d_{3}\right)^{3}[[X-Y,[X, Y]], X]- \\
\frac{1}{12}\left(d_{1}+d_{2}+d_{3}\right)^{3}[X+Y,[[X, Y], X]]- \\
-\frac{1}{2}\left(d_{1}+d_{2}+d_{3}\right)^{2}[X+Y,[Y, X]]- \\
\frac{1}{4}\left(d_{1}+d_{2}+d_{3}\right)^{3}[X+Y,[[X, Y], X]]- \\
\frac{1}{6}\left(d_{1}+d_{2}+d_{3}\right)^{3}[X+Y,[X+Y,[Y, X]]] \\
\frac{1}{12}\left(d_{1}+d_{2}+d_{3}\right)^{3}[X+Y,[X+Y,[Y, X]]]
\end{array}\right)+ \\
\exp d_{4}\left\{\begin{array}{c}
-\frac{1}{2}\left(d_{1}+d_{2}+d_{3}\right)[Y, X]-\frac{1}{4}\left(d_{1}+d_{2}+d_{3}\right)^{2}[[X, Y], X]- \\
\frac{1}{6}\left(d_{1}+d_{2}+d_{3}\right)^{2}[X+Y,[Y, X]]+ \\
-\frac{1}{2}\left(d_{1}+d_{2}+d_{3}\right)^{2}[X+Y,[Y, X]]- \\
\frac{1}{4}\left(d_{1}+d_{2}+d_{3}\right)^{3}[X+Y,[[X, Y], X]]- \\
\frac{1}{6}\left(d_{1}+d_{2}+d_{3}\right)^{3}[X+Y,[X+Y,[Y, X]]] \\
\frac{1}{12}\left(d_{1}+d_{2}+d_{3}\right)^{3}[X+Y,[X+Y,[Y, X]]]
\end{array}\right\} .
\end{gathered}
$$

$\exp \left(d_{1}+d_{2}+d_{3}\right)(X+Y)+\frac{1}{2}\left(d_{1}+d_{2}+d_{3}\right)^{2}[X, Y]+$ $\frac{1}{12}\left(d_{1}+d_{2}+d_{3}\right)^{3}[X-Y,[X, Y]]+$ $d_{4}\left\{\begin{array}{c}X+Y+\frac{1}{2}\left(d_{1}+d_{2}+d_{3}\right)[X, Y]+\frac{1}{4}\left(d_{1}+d_{2}+d_{3}\right)^{2}[[X, Y], Y]- \\ \frac{1}{6}\left(d_{1}+d_{2}+d_{3}\right)^{2}[X+Y,[X, Y]]+ \\ \frac{1}{4}\left(d_{1}+d_{2}+d_{3}\right)^{2}[X+Y,[X, Y]]\end{array}\right\}$. $\exp d_{4}\left\{\begin{array}{c}\frac{1}{24}\left(d_{1}+d_{2}+d_{3}\right)^{3}[[X-Y,[X, Y]], Y]- \\ \frac{1}{12}\left(d_{1}+d_{2}+d_{3}\right)^{3}[X+Y,[[X, Y], Y]]+ \\ \frac{1}{4}\left(d_{1}+d_{2}+d_{3}\right)^{3}[X+Y,[[X, Y], Y]]- \\ \left.\frac{1}{6}\left(d_{1}+d_{2}+d_{3}\right)^{3}[X+Y,[X+Y,[X, Y]]]-\right)^{3}[X,[X, Y]]+ \\ \frac{1}{12}\left(d_{1}+d_{2}+d_{3}\right)^{3}[X+Y,[X+Y,[X]] \\ \frac{1}{8}\left(d_{1}+d_{2}+d_{3}\right)^{3}[X+Y,[X+Y,[X, Y]]]\end{array}\right\}$.

We go on once more

$$
\begin{aligned}
& =\exp d_{4}\left\{\begin{array}{c}
-\frac{1}{24}\left(d_{1}+d_{2}+d_{3}\right)^{3}[[X-Y,[X, Y]], X]- \\
\frac{1}{12}\left(d_{1}+d_{2}+d_{3}\right)^{3}[X+Y,[[X, Y], X]]- \\
-\frac{1}{2}\left(d_{1}+d_{2}+d_{3}\right)^{2}[X+Y,[Y, X]]- \\
\frac{1}{2}\left(d_{1}+d_{2}+d_{3}\right)^{3}[X+Y,[[X, Y], X]]- \\
\frac{1}{6}\left(d_{1}+d_{2}+d_{3}\right)^{3}[X+Y,[X+Y,[Y, X]]] \\
\frac{1}{12}\left(d_{1}+d_{2}+d_{3}\right)^{3}[X+Y,[X+Y,[Y, X]]]
\end{array}\right\} . \\
& \exp \left(d_{1}+d_{2}+d_{3}\right)(X+Y)+\frac{1}{2}\left(d_{1}+d_{2}+d_{3}\right)^{2}[X, Y]+ \\
& \frac{1}{12}\left(d_{1}+d_{2}+d_{3}\right)^{3}[X-Y,[X, Y]]+
\end{aligned}
$$




$$
\begin{gathered}
d_{4}\left\{\begin{array}{c}
X+Y+\frac{1}{2}\left(d_{1}+d_{2}+d_{3}\right)[X, Y]+\frac{1}{4}\left(d_{1}+d_{2}+d_{3}\right)^{2}[[X, Y], Y]- \\
\frac{1}{6}\left(d_{1}+d_{2}+d_{3}\right)^{2}[X+Y,[X, Y]]+ \\
\frac{1}{4}\left(d_{1}+d_{2}+d_{3}\right)^{2}[X+Y,[X, Y]]- \\
\frac{1}{6}\left(d_{1}+d_{2}+d_{3}\right)^{2}[X+Y,[Y, X]] \\
\frac{1}{2}\left(d_{1}+d_{2}+d_{3}\right)[Y, X]-\frac{1}{4}\left(d_{1}+d_{2}+d_{3}\right)^{2}[[X, Y], X]- \\
\exp d_{4}\left\{\begin{array}{c}
\frac{1}{24}\left(d_{1}+d_{2}+d_{3}\right)^{3}[[X-Y,[X, Y]], Y]- \\
\frac{1}{12}\left(d_{1}+d_{2}+d_{3}\right)^{3}[X+Y,[[X, Y], Y]]+ \\
\frac{1}{4}\left(d_{1}+d_{2}+d_{3}\right)^{3}[X+Y,[[X, Y], Y]]- \\
\frac{1}{6}\left(d_{1}+d_{2}+d_{3}\right)^{3}[X+Y,[X+Y,[X, Y]]] \\
\frac{1}{12}\left(d_{1}+d_{2}+d_{3}\right)^{3}[X+Y,[X+Y,[X, Y]]]+ \\
\frac{1}{8}\left(d_{1}+d_{2}+d_{3}\right)^{3}[X+Y,[X+Y,[X, Y]]]
\end{array}\right\}
\end{array}\right\}
\end{gathered}
$$

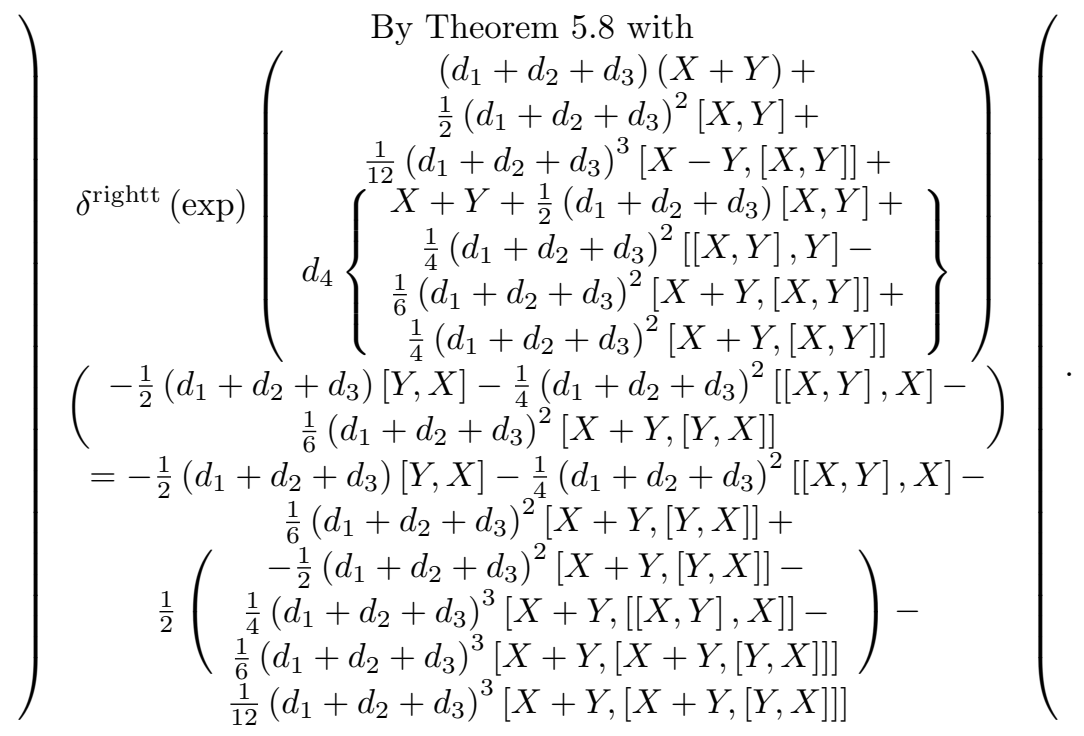

We go on once more

$$
\begin{gathered}
=\exp d_{4}\left\{\begin{array}{c}
-\frac{1}{24}\left(d_{1}+d_{2}+d_{3}\right)^{3}[[X-Y,[X, Y]], X]- \\
\frac{1}{12}\left(d_{1}+d_{2}+d_{3}\right)^{3}[X+Y,[[X, Y], X]]- \\
-\frac{1}{4}\left(d_{1}+d_{2}+d_{3}\right)^{3}[X+Y,[[X, Y], X]]- \\
\frac{1}{6}\left(d_{1}+d_{2}+d_{3}\right)^{3}[X+Y,[X+Y,[Y, X]]] \\
\frac{1}{12}\left(d_{1}+d_{2}+d_{3}\right)^{3}[X+Y,[X+Y,[Y, X]]]- \\
\frac{1}{8}\left(d_{1}+d_{2}+d_{3}\right)^{3}[X+Y,[X+Y,[Y, X]]]
\end{array}\right\} . \\
\exp d_{4}\left\{\begin{array}{c}
\frac{1}{4}\left(d_{1}+d_{2}+d_{3}\right)^{2}[X+Y,[Y, X]]+ \\
\frac{1}{8}\left(d_{1}+d_{2}+d_{3}\right)^{3}[X+Y,[X+Y,[Y, X]]]
\end{array}\right\} . \\
\exp \left(d_{1}+d_{2}+d_{3}\right)(X+Y)+\frac{1}{2}\left(d_{1}+d_{2}+d_{3}\right)^{2}[X, Y]+ \\
\frac{1}{12}\left(d_{1}+d_{2}+d_{3}\right)^{3}[X-Y,[X, Y]]+
\end{gathered}
$$


THE BAKER-CAMPBELL-HAUSDORFF FORMULA AND ...

$$
\begin{aligned}
& d_{4}\left\{\begin{array}{c}
X+Y+\frac{1}{2}\left(d_{1}+d_{2}+d_{3}\right)[X, Y]+\frac{1}{4}\left(d_{1}+d_{2}+d_{3}\right)^{2}[[X, Y], Y]- \\
\frac{1}{6}\left(d_{1}+d_{2}+d_{3}\right)^{2}[X+Y,[X, Y]]+ \\
\frac{1}{4}\left(d_{1}+d_{2}+d_{3}\right)^{2}[X+Y,[X, Y]]- \\
\frac{1}{2}\left(d_{1}+d_{2}+d_{3}\right)[Y, X]-\frac{1}{4}\left(d_{1}+d_{2}+d_{3}\right)^{2}[[X, Y], X]- \\
\frac{1}{6}\left(d_{1}+d_{2}+d_{3}\right)^{2}[X+Y,[Y, X]]
\end{array}\right\} . \\
& \exp d_{4}\left\{\begin{array}{c}
\frac{1}{24}\left(d_{1}+d_{2}+d_{3}\right)^{3}[[X-Y,[X, Y]], Y]- \\
\frac{1}{12}\left(d_{1}+d_{2}+d_{3}\right)^{3}[X+Y,[[X, Y], Y]]+ \\
\frac{1}{4}\left(d_{1}+d_{2}+d_{3}\right)^{3}[X+Y,[[X, Y], Y]]- \\
\frac{1}{6}\left(d_{1}+d_{2}+d_{3}\right)^{3}[X+Y,[X+Y,[X, Y]]]- \\
\frac{1}{12}\left(d_{1}+d_{2}+d_{3}\right)^{3}[X+Y,[X+Y,[X, Y]]]+ \\
\frac{1}{8}\left(d_{1}+d_{2}+d_{3}\right)^{3}[X+Y,[X+Y,[X, Y]]]
\end{array}\right\} .
\end{aligned}
$$

We go on once more

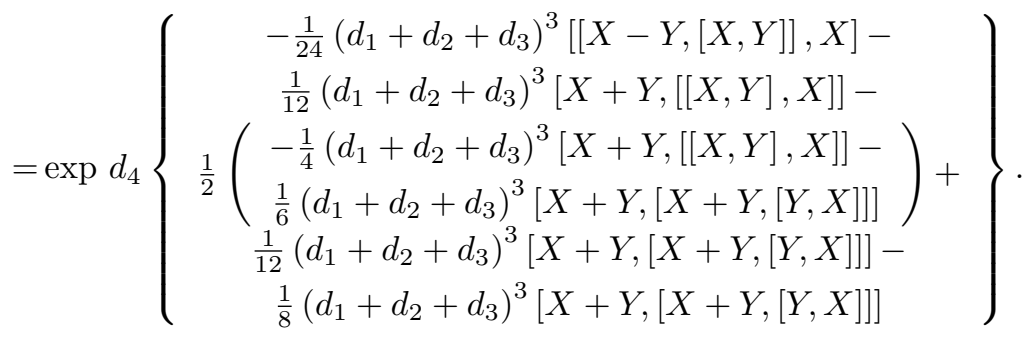

$\exp \left(d_{1}+d_{2}+d_{3}\right)(X+Y)+\frac{1}{2}\left(d_{1}+d_{2}+d_{3}\right)^{2}[X, Y]+$ $\frac{1}{12}\left(d_{1}+d_{2}+d_{3}\right)^{3}[X-Y,[X, Y]]+$

$d_{4}\left\{\begin{array}{c}X+Y+\frac{1}{2}\left(d_{1}+d_{2}+d_{3}\right)[X, Y]+\frac{1}{4}\left(d_{1}+d_{2}+d_{3}\right)^{2}[[X, Y], Y]- \\ \frac{1}{6}\left(d_{1}+d_{2}+d_{3}\right)^{2}[X+Y,[X, Y]]+ \\ \frac{1}{4}\left(d_{1}+d_{2}+d_{3}\right)^{2}[X+Y,[X, Y]]- \\ \frac{1}{2}\left(d_{1}+d_{2}+d_{3}\right)[Y, X]-\frac{1}{4}\left(d_{1}+d_{2}+d_{3}\right)^{2}[[X, Y], X]- \\ \frac{1}{6}\left(d_{1}+d_{2}+d_{3}\right)^{2}[X+Y,[Y, X]]+ \\ \frac{1}{4}\left(d_{1}+d_{2}+d_{3}\right)^{2}[X+Y,[Y, X]]\end{array}\right\}$.

$$
\exp d_{4}\left\{\begin{array}{c}
\frac{1}{24}\left(d_{1}+d_{2}+d_{3}\right)^{3}[[X-Y,[X, Y]], Y]- \\
\frac{1}{12}\left(d_{1}+d_{2}+d_{3}\right)^{3}[X+Y,[[X, Y], Y]]+ \\
\frac{1}{4}\left(d_{1}+d_{2}+d_{3}\right)^{3}[X+Y,[[X, Y], Y]]- \\
\left.\frac{1}{6}\left(d_{1}+d_{2}+d_{3}\right)^{3}[X+Y,[X+Y,[X, Y]]]-\right) \\
\frac{1}{12}\left(d_{1}+d_{2}+d_{3}\right)^{3}[X+Y,[X+Y,[X, Y]]]+ \\
\frac{1}{8}\left(d_{1}+d_{2}+d_{3}\right)^{3}[X+Y,[X+Y,[X, Y]]]
\end{array}\right\}
$$




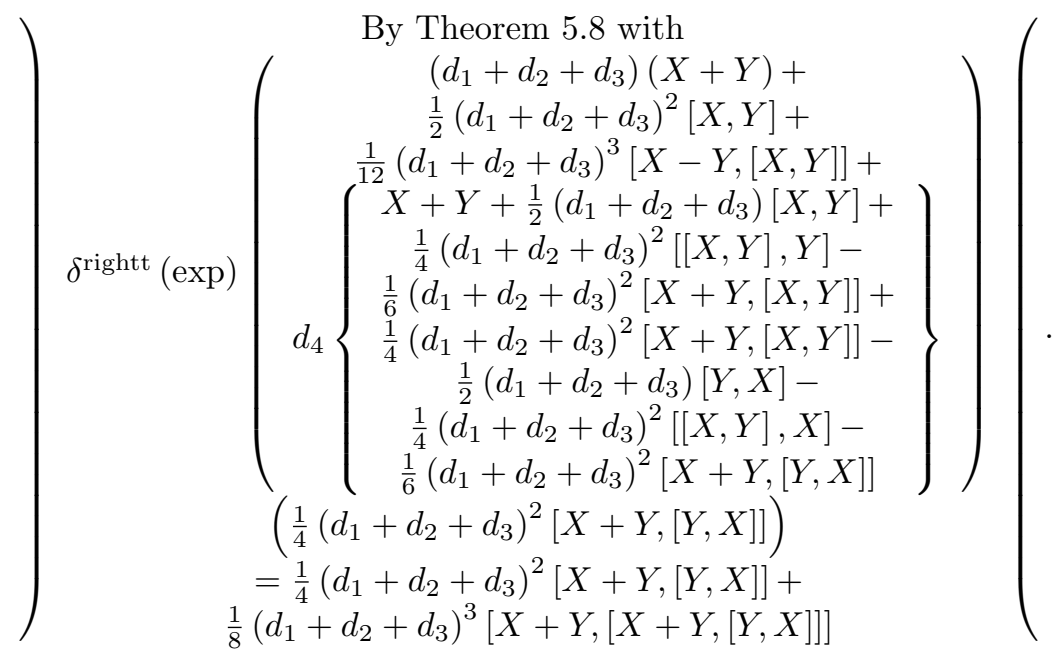

We go on once more

$$
\begin{aligned}
& =\exp \left(d_{1}+d_{2}+d_{3}\right)(X+Y)+\frac{1}{2}\left(d_{1}+d_{2}+d_{3}\right)^{2}[X, Y]+ \\
& \frac{1}{12}\left(d_{1}+d_{2}+d_{3}\right)^{3}[X-Y,[X, Y]]+ \\
& d_{4}\left\{X+Y+\left(d_{1}+d_{2}+d_{3}\right)[X, Y]+\frac{1}{4}\left(d_{1}+d_{2}+d_{3}\right)^{2}[X-Y,[X, Y]]\right\} \text {. } \\
& \exp d_{4}\left(d_{1}+d_{2}+d_{3}\right)^{3}\left\{\begin{array}{c}
\frac{1}{24}[X,[X-Y,[X, Y]]]+ \\
\left(\frac{1}{12}-\frac{1}{8}\right)[X+Y,[X,[X, Y]]]+ \\
\left(\frac{1}{12}+\frac{1}{12}-\frac{1}{8}\right)[X+Y,[X+Y,[Y, X]]]- \\
\frac{1}{24}[Y,[X-Y,[X, Y]]]+ \\
\left(\frac{1}{12}-\frac{1}{8}\right)[X+Y,[Y,[X, Y]]]- \\
\left(\frac{1}{12}+\frac{1}{12}-\frac{1}{8}\right)[X+Y,[X+Y,[X, Y]]]
\end{array}\right\} \\
& =\exp \left(d_{1}+d_{2}+d_{3}+d_{4}\right)(X+Y)+\frac{1}{2}\left(d_{1}+d_{2}+d_{3}+d_{4}\right)^{2}[X, Y]+ \\
& \frac{1}{12}\left(d_{1}+d_{2}+d_{3}+d_{4}\right)^{3}[X-Y,[X, Y]] \text {. } \\
& \exp d_{4}\left(d_{1}+d_{2}+d_{3}\right)^{3}\left\{\begin{array}{c}
-\frac{1}{24}([X,[Y,[X, Y]]]+[Y,[X,[X, Y]]])+ \\
\frac{1}{24}[X+Y,[X+Y,[Y, X]]]- \\
-\frac{1}{24}([X,[Y,[X, Y]]]+[Y,[X,[X, Y]]])- \\
\frac{1}{24}[X+Y,[X+Y,[X, Y]]]
\end{array}\right\} \\
& =\exp \left(d_{1}+d_{2}+d_{3}+d_{4}\right)(X+Y)+\frac{1}{2}\left(d_{1}+d_{2}+d_{3}+d_{4}\right)^{2}[X, Y]+ \\
& \frac{1}{12}\left(d_{1}+d_{2}+d_{3}+d_{4}\right)^{3}[X-Y,[X, Y]] \text {. } \\
& \exp -\frac{1}{12} d_{4}\left(d_{1}+d_{2}+d_{3}\right)^{3}\left(\begin{array}{c}
{[X,[Y,[X, Y]]]+[Y,[X,[X, Y]]]+} \\
{[X+Y,[X+Y,[X, Y]]]}
\end{array}\right) \\
& =\exp \left(d_{1}+d_{2}+d_{3}+d_{4}\right)(X+Y)+\frac{1}{2}\left(d_{1}+d_{2}+d_{3}+d_{4}\right)^{2}[X, Y]+
\end{aligned}
$$




$$
\begin{aligned}
& \frac{1}{12}\left(d_{1}+d_{2}+d_{3}+d_{4}\right)^{3}[X-Y,[X, Y]]- \\
& \frac{1}{48}\left(d_{1}+d_{2}+d_{3}+d_{4}\right)^{4}\left(\begin{array}{c}
{[X,[Y,[X, Y]]]+[Y,[X,[X, Y]]]+} \\
{[X+Y,[X+Y,[X, Y]]]}
\end{array}\right) .
\end{aligned}
$$

\section{REFERENCES}

[1] H.F. Baker, Alternants and continuous groups, Proceedings of the London Mathematical Society II 3 (1905), 24-47.

[2] J. E. Campbell, On a law of combination of operators bearing on the theory of continuous transformation groups, Proceedings of the London Mathematical Society 28 (1897), 381390.

[3] J.E. Campbell, On a law of combination of operators (second paper), Proceedings of the London Mathematical Society 29 (1898), 14-32.

[4] F. Fer, Résolution de l'équation matricielle $d U / d t=p U$ par produit infini d'éxponentielles matricielles, Académie Royale de Belgique de la Classe des Sciences V 44 (1958), 818-829.

[5] F. Hausdorff, Die symbolische Exponential Formel in der Gruppentheorie, Berichte über die Verhandlungen Sächsischen Akademie der Wisssenchaften zu Leipzig 58 (1906), 19-48.

[6] A. Kock, Taylor series calculus for ring objects of line type, J. Pure Appl. Algebra 12 (1978), 271-293.

[7] A. Kriegl, P. W. and Michor, The Convenient Setting of Global Analysis, American Mathematical Society, Rhode Island, 1997.

[8] R. Lavendhomme, Basic Concepts of Synthetic Differential Geometry, Kluwer Academic Publishers, Dordrecht, 1996.

[9] W. Magnus, On the exponential solution of differential equations for a linear operator, Communications on Pure and Applied Mathematics 7 (1954), 649-673.

[10] P. W. Michor, Topics in Differential Geometry, American Mathematical Society, Rhode Island, 2008.

[11] H. Omori, Y. Maeda and A. Yoshioka, On regular Fréchet Lie groups I. Some differential geometric expressions of Fourier integral operators on a Riemannian manifolds, Tokyo Journal of Mathematics 3 (1980), 353-390.

[12] H. Omori, Y. Maeda and A. Yoshioka, On regular Fréchet Lie groups II. Composition rules of Fourier integral operators on a Riemannian manifold, Tokyo Journal of Mathematics 4 (1981), 221-253.

[13] H. Omori, Y. Maeda and A. Yoshioka, On regular Fréchet Lie groups III, Tokyo Journal of Mathematics 4 (1981), 255-277.

[14] H. Omori, Y. Maeda and A. Yoshioka, On regular Fréchet Lie groups IV. Definitions and fundamental theorems, Tokyo Journal of Mathematics 5 (1982), 365-398.

[15] H. Omori, Y. Maeda and A. Yoshioka, On regular Fréchet Lie groups V. Several basic properties, Tokyo Journal of Mathematics 6 (1983), 39-64.

[16] H. Omori, Y. Maeda and A. Yoshioka, On regular Fréchet Lie groups VI. Infinite dimensional Lie groups which appear in general relativity, Tokyo Journal of Mathematics 6 (1983), 217-246.

[17] V.S. Varadarajan, Lie Groups, Lie Algebras, and their Representations, Prentice-Hall, Englewood Cliffs, New Jersey, 1974.

[18] H. Zassenhaus, Über Lieschen Ringe mit Primzahlcharakteristik, Abhandlungen aus dem Mathematischen Seminar der Universität Hamburg 13 (1939), 1-100.

Hirokazu Nishimura, Institute of Mathematics, University of Tsukuba, Tsukuba, Ibaraki, 305-8571, Japan

e-mail: logic@math.tsukuba.ac.jp 
